\title{
Mass spectrometry and its role in advancing cluster science
}

\author{
P. Jena,
}

Department of Physics, Virginia Commonweal University, Richmond, Virginia 23284-2000

\author{
A.W. Castleman, Jr., \\ Departments of Physics and Chemistry, The Pennsylvania State University, University Park, \\ Pennsylvania 16802
}

\begin{abstract}
Clusters composed of a few to a few hundred atoms are the ultimate nanoparticles where every atom and every electron count. Over the past 50 years clusters have evolved as a new field of matter intermediate between atoms and bulk. In this retrospective we trace the role mass spectrometry has played in this emerging field with emphasis on how extremely intense mass peaks indicating a high abundance of clusters led to the discovery of magic numbers in alkali metal clusters, the fullerenes in carbon clusters, and "met-cars" in transition-metal carbide clusters. Magic numbers in alkali metal clusters in turn helped bridge the gap between two disparate fields, atomic physics and nuclear physics. Studies of mass spectra combined with theory and a variety of other experimental techniques have since led to a fundamental understanding of the structure-property relationships of clusters, thus broadening the scope of cluster science. Clusters not only serve as a bridge between atoms and bulk and between atomic and nuclear physics, but also as a bridge across many disciplines. Although this retrospective is focused on atomic clusters, briefly discussed is the solvation phenomenon, a subject to which mass spectrometry has and continues to make a large contribution.
\end{abstract}




\section{Introduction:}

The influence of size and dimensionality on the behavior of matter can be enormous. Indeed, due to quantum confinement of electrons brought about by finite size, particles of nano-meter dimensions can and do exhibit remarkable properties not seen in their bulk phase. However, a fundamental understanding of why and how these properties emerge has not been easy since it is difficult to control the size, shape, and composition of nano-particles, one atom at a time. The emergence of cluster science in the past 50 years has changed that paradigm. At the root of this evolution lies the contribution of mass spectroscopy which has made it possible to isolate clusters with specific mass. Thus, atomic clusters are the ultimate nano-particles whose composition and size is often known or determinable with atomic precision.

This article traces a few of the many discoveries in cluster science that have been possible because of our ability to measure the relative abundance of clusters and relate the pronounced peaks in the mass spectra to their thermodynamic stability and underlying electronic structure. In other situations, kinetic factors govern the emerging properties. As time progressed from roughly half a century ago, cluster science emerged as a major scientific field [1-4], enabled in large measure to the advent of various advanced mass spectrometers. The world has now come to realize some of the dreams that Richard Feynman had in late 1950's that "there is plenty of room at the bottom".

Although the exact date of discovery of canal rays (rays of positive charge) is not settled, their study in the late 1890 's, when subject to strong electric and magnetic fields, is certainly a forerunner to the development of mass spectrometry. As elsewhere discussed in this book, mass spectrometry is marked by its 100 year birthday in 2014. With all of the many ion sources that have been developed over the century, rarely are materials found that cannot be analyzed via 
mass spectrometry, once some clever technique is utilized to introduce them into the gas phase. The exceptions usually are ones that readily fragment when brought into the gas phase; these generally require special techniques to introduce them into a mass spectrometer such as via matrix desorption/vaporization or in favorable cases using electrospray methods.

Herein we turn our attention to the field of cluster science, a very broad subject which serves as an example for the investigation of how properties of matter evolve as a function of size from a single atom to bulk materials. One of the approaches in cluster science has been to produce clusters via an expansion method (such as adiabatic condensation) and then interrogating the resulting cluster distribution via mass spectrometry. A common though not always correct assumption is that the resulting distribution identifies bonded systems. More about bonding and stability is discussed later.

Although not quantitative, it has become common to categorize systems into matter composed of soft and hard constituents. Researchers who were particularly interested in the characteristics of beam dynamics dominated early activities in the field. Numerous studies have been reported for studies made with clusters comprised of noble gas species, others of systems composed of van der Waal's bonded elements, simple metals, and eventually transition metals and main group elements. Relative stabilities of clusters and in particular those exhibiting pronounced peaks in the mass spectra (commonly referred to as magic numbers) are interpreted in terms of electron counting rules; electron shell closure rule based on the jellium model for simple metals, octet rule for elements with atomic number less than 20, 18-electron rule for transition metals, Wade-Mingos rule for electron deficient systems, and aromatic rule for organic and in some cases even inorganic systems. In clusters composed of atoms with closed electronic 
shells such as noble gas atoms, it is the close atomic packing that dictates their enhanced stability and hence their abundance.

As mentioned before, cluster science has developed into a major field. Our intention here is not to cover all the seminal works that have appeared in this field, but instead we focus only on atomic clusters with different bonding characteristics, paying particular attention to the role mass spectrometry has played in elucidating their stability, geometry and underlying electronic structure. Even for each system we discuss, there are numerous papers that have been published and it is not possible for us to cite them all. Instead we only refer to some representative papers and ask the reader to search the literature for a comprehensive list [5].

\section{Mass spectrometry and cluster bonding}

The first step in unraveling the structure-property relationships of atomic clusters (or analogous molecular species) is to understand their geometry and thermodynamic stability. Seldom are there experimental techniques available that can directly probe the geometries of clusters. And when they are available they involve methods suitable only for very small cluster sizes, yielding information on their relative stabilities from the peak intensities of their mass spectra. Note that the stability of a cluster is governed by both the electronic structure and atomic packing; hence, mass spectrometry can indirectly shed light on the atomic and electronic structure of clusters. Although it must be established individually in each case, generally the most pronounced peaks in the mass spectrum of a cluster distribution relate to the most stable clusters which are referred to as magic numbers.

In the early days of mass spectrometry attention was paid primarily to molecular clusters and clusters of low melting point metals, simply because it was easy to produce them. The advent of laser vaporization technique [6] as well as pulsed arc cluster ion source (PACIS) [7] 
allowed researchers to study clusters of high melting point elements and much of the rich physics and chemistry we know about clusters have emerged since then. Although in this retrospective we focus on structure and bonding of atomic clusters, we begin with a brief discussion of water clusters where the size distribution and growth in the gas phase of ions in $\mathrm{H}^{+}\left(\mathrm{H}_{2} \mathrm{O}\right)_{n}(n \leq 28)$ series by Searcy and Fenn [8] gave the first indication of magic number at $n=21$. Yang and Castleman [9] later extended this study up to $n=60$ and observed magic numbers (i.e. clusters showing pronounced peaks in the mass spectra) at $n=21,24,26$, and 28. In Fig. 1 we present the observed mass spectra by these authors. A synergistic study by Duncan and coworkers [10] involving infrared spectroscopy experiment and calculations of lowest-energy structures of $\mathrm{H}^{+}\left(\mathrm{H}_{2} \mathrm{O}\right)_{n}$ provided direct evidence of the structure of the magic number cluster $(n=21)$ where all the dangling $\mathrm{OH}$ groups were found to arise from water molecules in similar bonding sites.

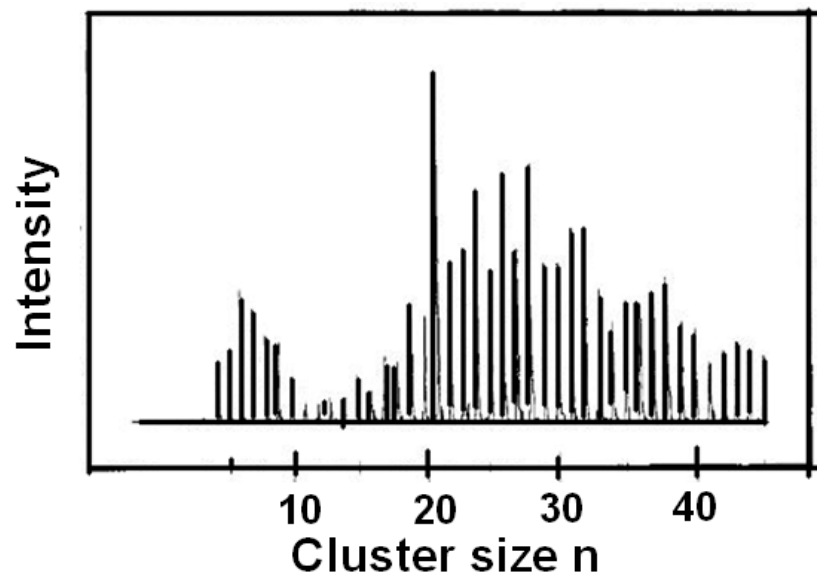

Fig. 1. Mass spectrum of protonated water clusters $\mathrm{H}^{+}\left(\mathrm{H}_{2} \mathrm{O}\right)_{n}(n=4-45)$ at $110 \mathrm{~K}$ flow tube temperature and 0.3 Torr He. [Ref. 9] 
In the following we focus on magic numbers in atomic clusters and show how, under favorable circumstances, they have enabled not only an understanding of the origin of cluster stability but also of their underlying atomic and electronic structure.

\section{A. Clusters of noble gas atoms - van der Waal's bonding}

We begin with the simplest class of clusters, namely those composed of noble gas atoms

$(\mathrm{He}, \mathrm{Ne}, \mathrm{Ar}, \mathrm{Xe}, \mathrm{Kr}, \mathrm{Rn})$. These atoms are characterized by closed $n \mathrm{~s}^{2} n \mathrm{p}^{6}$ electronic shells, hence they are chemically inert; the bonding between the atoms is weak and governed by van der Waal's interaction. Electronic structure, therefore, does not play an important role in determining their cluster geometries. The noble gas atoms can be described in terms of a hard sphere packing model where space filling is the only requirement to minimize cluster's energy. One such packing is given by MacKay icosahedra where $13,55,147, \ldots$ atoms are needed to fill successive shells of an icosahedron. This geometric shell closure leads to enhanced stability of these clusters which then should reflect in the mass intensity distribution. This is, indeed, what was observed by Recknagel and coworkers [11]. In Fig. 2 we reproduce the mass spectra of Xe clusters taken by these authors who have identified pronounced peaks at 13, 19, 25, 55, 71, 87 and 147. Note that the intensities of peaks drop abruptly when one extra atom is added to the above magic numbers. The agreement of the magic numbers in Fig. 2 with the packing of MacKay icosahedra confirms that atomic-shell closure is the driving force for the formation of magic clusters of noble gas atoms. In order to make an unequivocal correlation, a mechanism which accounts for an increase in stable cluster intensities must be identified. 


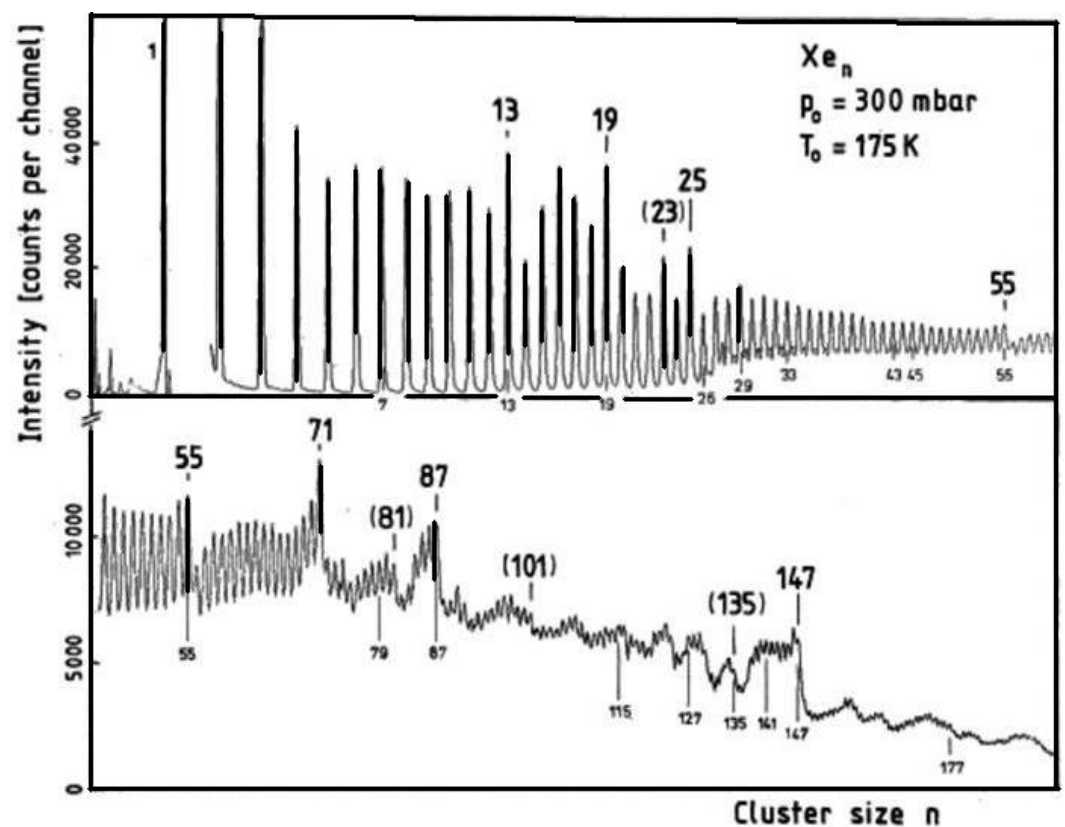

Fig.2. Mass spectrum of xenon clusters. Observed magic numbers are marked in bold face; brackets are used for numbers with less pronounced effects. Numbers below the curve indicate predictions or distinguish sphere packings [11].

Fragmentation of multiply charged clusters can also illustrate their underlying electronic structure and bonding features. As a cluster becomes multiply ionized, the positive charges repel each other and eventually move to the farthest end of a cluster to minimize their Coulomb repulsion energy. If this repulsive energy is larger than the binding energy of the cluster it explodes into small fragments. Thus, the critical size of a cluster before Coulomb explosion takes place can provide a signature of the relative strength of their binding. Consider, for example, three different kinds of clusters; clusters of noble gas atoms such as Xe that are bonded by van der Waal's interaction and weak, clusters of NaI where the bonding is ionic and strong, and clusters of Pd where the bonding is metallic and intermediate. Thus, the critical size for Coulomb explosion should be largest for Xe clusters and smallest for $\mathrm{NaI}$ clusters with $\mathrm{Pb}$ clusters being in 
the middle. This is, indeed, what was observed by Recknagel and coworkers [12] who found the critical size for Coulomb explosion of doubly charged $\mathrm{Xe}, \mathrm{Pb}$, and $\mathrm{NaI}$ clusters to be 52, 30, and 20 respectively. Direct fragmentation or association ionization may also compete as has been observed.

\section{B. Clusters of simple metals- Electronic shell closure rule based on the jellium model}

Clusters of simple metals such as alkali and alkaline earth metals and aluminum lie at the other extreme of the van der Waal's bonded noble gas clusters. Here, electrons are nearly free and according to the jellium model move in a constant potential provided by a uniform distribution of positive charge of the ions. The total energies of these clusters can be calculated easily [13] by confining the valence electrons in an infinite spherical potential well whose radius is determined by the size of the clusters. The quantized energy levels of such a potential well can be identified by their orbital angular momentum quantum numbers $1 \mathrm{~S}^{2}, 1 \mathrm{P}^{6}, 1 \mathrm{D}^{10}, 2 \mathrm{~S}^{2}, 1 \mathrm{~F}^{14}, 2 \mathrm{P}^{6}$, etc. Clusters that contain just enough electrons to close these electronic shells are expected to be more stable than their neighboring species just as noble gas atoms, due to their closed electronic shells, are chemically inert. Consider, for example, $\mathrm{Na}$ clusters. Since $\mathrm{Na}$ is monovalent, the number of electrons in a $\mathrm{Na}$ cluster equals the number of $\mathrm{Na}$ atoms. Thus, clusters containing 2, $8,20,40, \ldots \mathrm{Na}$ atoms should be more stable than their neighbors and peaks corresponding to these numbers in mass spectra should be more pronounced. This is exactly what was observed by Knight and coworkers [13]. Their results are reproduced in Fig. 3. The second derivative of the total energies of these clusters is plotted in Fig. 3(b). Peaks in Fig. 3(b) signify cluster stability. We note that the pronounced peaks at $\mathrm{N}=2,8,20,40, \ldots$ in Fig. 3(a) are in perfect agreement with the jellium model predictions in Fig. 3(b). Thus, one can conclude that the relative stability of clusters of nearly free electron metals is governed by electronic shell closure rule. This 
pioneering work, in addition to describing the origin of the stability of free electron metal clusters, provided a link to nuclear physics. The authors noted that nuclei with nucleon numbers of $2,8,20,40, \ldots$ were found to be very stable and the origin of this stability was explained by nuclear shell closure. That atomic clusters governed by Coulomb forces and nuclei bound by nuclear forces can exhibit similar stability pattern provided a bridge between atomic and nuclear physics; and importantly, in the context of the present chapter the bridge to mass and charge, and hence mass spectrometry, is also evident. Aspects such as fission and giant diploe resonances [14] well known in nuclei were later explored in atomic clusters and the synergy between cluster scientists and nuclear physicists have enriched both disciplines [15].

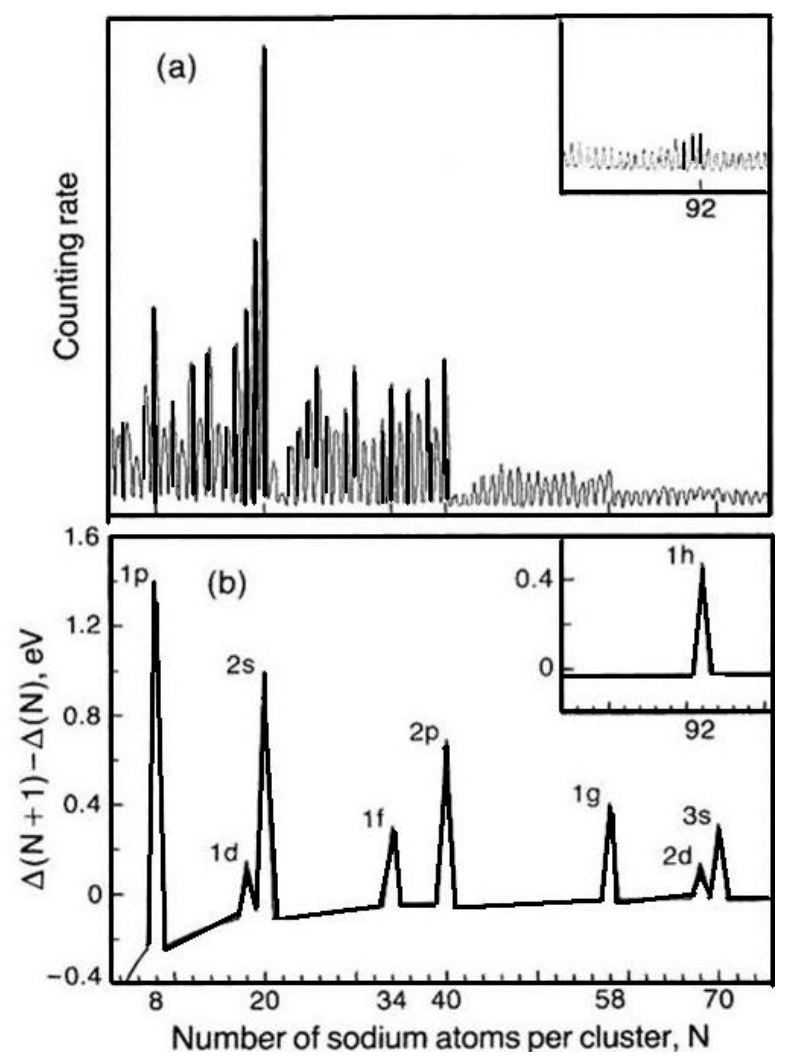

Fig. 3. (a) Mass spectrum of sodium clusters, $N=4-75$. The inset corresponds to $N=75-100$. (b) The calculated change in the electronic energy difference, $\Delta(\mathrm{N}+1)-\Delta(\mathrm{N})$ vs $\mathrm{N}$. The labels and the peaks correspond to the closed-shell orbitals. [Ref. 13] 
As noted above, stability of noble atom clusters is governed by atomic shell closure while stability of clusters of free-electron metals is governed by electronic shell closure. The question explored by Martin and coworkers [16] was whether atomic shell closure could ever play a role in the stability of $\mathrm{Na}$ clusters. The authors measured the mass spectra of $\mathrm{Na}$ clusters containing up to 22000 atoms. For small clusters containing less than $1500 \mathrm{Na}$ toms, Martin and coworkers observed the magic numbers consistent with the electronic shell closure scheme in the jellium model. However, for larger clusters, they observed magic numbers consistent with completion of icosahedral and cuboctahedral shells of atoms. Their results are given in Fig. 4. The observed transition from electronic shell closure to atomic shell closure was attributed to be due to the mobility of atoms as a function of size. The authors hypothesized that very small clusters behave like liquid droplets and each time an atom is added it is absorbed into the droplet, maintaining a spherical-like shape. Large clusters, under experimental conditions, solidify and each newly added atom condenses onto the surface and remains there. Further growth takes place by the accumulation of shells of atoms. Manninen and Jena [17] subsequently estimated an upper limit for number of atoms in metal clusters capable of exhibiting shell structure by comparing the energy difference between the highest occupied and the lowest unoccupied state with crystal field splitting. The results indicated that electronic shell structures may persist in clusters containing as many as a million atoms. 


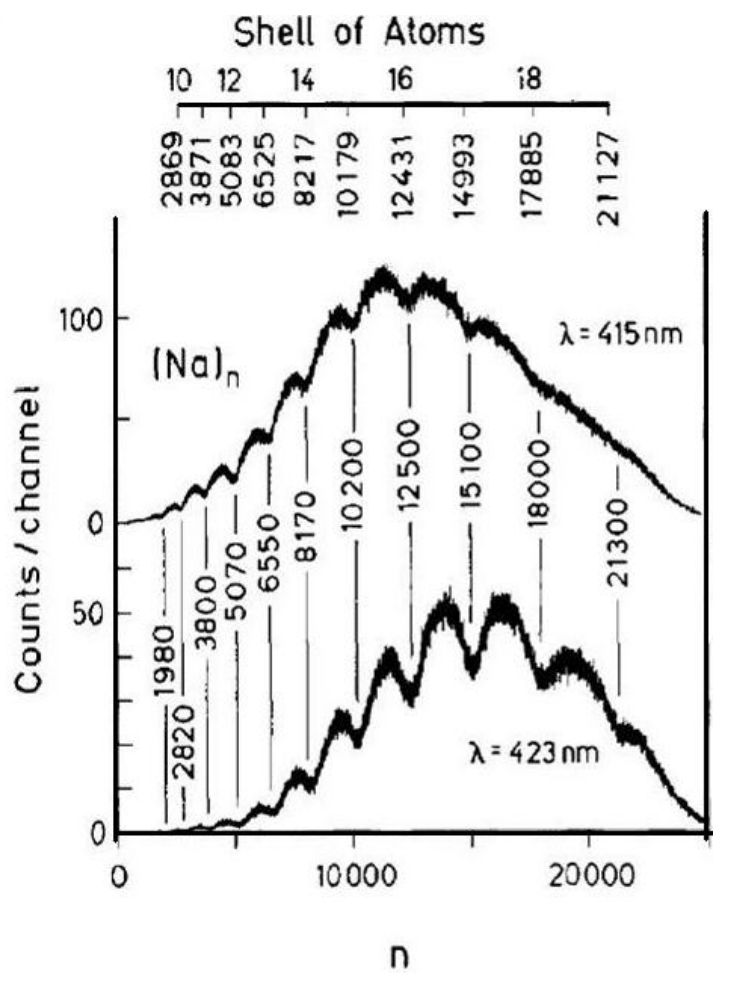

Fig. 4. Averaged mass spectra of $\mathrm{Na}_{n}$ clusters photoionized with 415 and $423 \mathrm{~nm}$ light. Well defined minima occur at values of $n$ corresponding to the total number of atoms in closed-packed cuboctahedra and nearly close-packed icosahedra (listed at top) [Ref. 16]

We note that clusters need to be charged in order to be mass isolated. The question then arises: are the pronounced peaks in the mass spectrum associated with the clusters that are most stable in the neutral or charged state? From the analysis provided above, it would appear that the magic peaks coprrespond to most stable neutral clusters. One can imagine in this case that clusters are born "neutral" and the most abunadant clusters are those that are most stable. What if conditions in the experimental set-up are such that clusters are "born" charged? What would the magic numbers be? Consider, for example, clusters are born in singly postively charged state. The jellium model would then predict [18] the magic numbers of singly ionized alkali metal clusters to shift by 1 , namely, clusters of $3,9,21,41, \ldots$ positively charged alkali atom clusters will be among the most stable species. The magic numbers will shift by 1 as the chrage on the 
cluster is successively increased (see Fig. 5). This is indeed what was oberved in the mass spectra of singly ionized $\mathrm{Rb}_{n}{ }^{+}$clusters [19] (see Fig. 6).

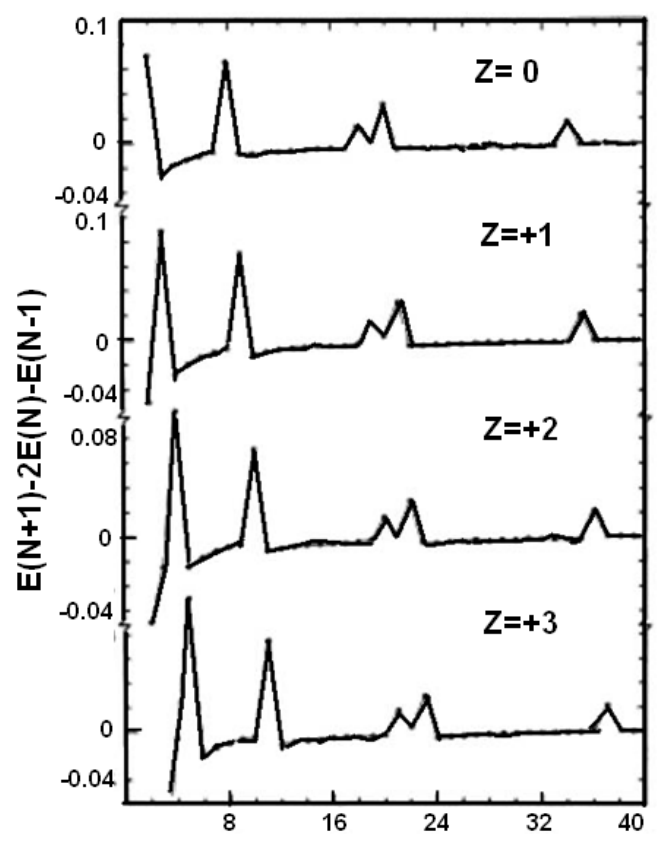

Fig. 5. Second derivative of the total energy $\left(\mathrm{E}_{\mathrm{N}+1}+\mathrm{E}_{\mathrm{N}-1}-2 \mathrm{E}_{\mathrm{N}}\right)$ (in Hartree units) as a function of $\mathrm{N}$ for $\mathrm{Li}_{\mathrm{N}}, \mathrm{Li}_{\mathrm{N}}^{+}$, $\mathrm{Li}_{\mathrm{N}}{ }^{2+}$, and $\mathrm{Li}_{\mathrm{N}}{ }^{3+}$. The results are based upon the jellium model. [Ref. 18 ]

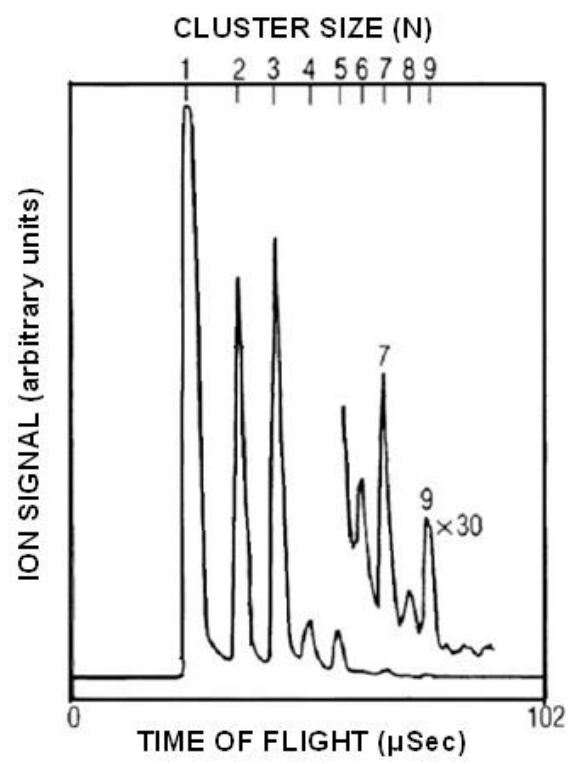

Fig. 6. Mass spectrum of $\mathrm{Rb}_{n}^{+}(\mathrm{n}=1-9)$ [Ref. 19] 
Another classic example where mass spectrometry of clusters has illustrated unique chemistry of charged clusters is that of negatively charged $\mathrm{Al}_{n}{ }^{-}$clusters [20]. Note that $\mathrm{Al}$ is trivalent, thus $\mathrm{Al}_{13}{ }^{-}$cluster containing 40 electrons should be a magic cluster due to electronic shell closure. The mass spectra taken by Castleman and coworkers [20] does indeed confirm this (see Fig. 7). Similarly, $\mathrm{Al}_{23}{ }^{-}$with 70 electrons also correspond to a magic cluster and this is confirmed from the pronounced peak in Fig. 7. However, the prominent peak of $\mathrm{Al}_{7}{ }^{-}$does pose an intersting question since with 22 electrons it should not constitute a magic cluster. Note that Jarrold et. al. [21] had earlier observed a predominent peak corresponding to $\mathrm{Al}_{7}^{+}$in the mass spectrum of ionized $\mathrm{Al}$ clusters (see Fig. 8). Since $\mathrm{Al}_{7}{ }^{+}$containes 20 electrons, the pronounced peak is consistent with the 20 -electron shell closure. The anomalous result of the prominent mass peak in $\mathrm{Al}_{7}{ }^{-}$was later shown, both experimenatlly [22] and theoretically [23], to be due to the multivalent nature of small Al clusters. Note that the large $3 s-3 p$ gap of the Al atom may allow $\mathrm{Al}$ to behave as a monovalent atom in small clusters, but trivalent in large clusters. When the cluster size becomes large, the $3 s$ and $3 p$ orbitals would overlap allowing Al to assume its normal trivalent state. The critical cluster size where transition from monovalent to trivalent $\mathrm{Al}$ is complete was shown to be around $n=7$ (see Fig. 9). Thus, $\mathrm{Al}_{7}{ }^{-}$where $\mathrm{Al}$ behaves as a monovalent species would contain 8 electrons, enough for $1 \mathrm{~S}^{2} 1 \mathrm{P}^{6}$ shell closing. 


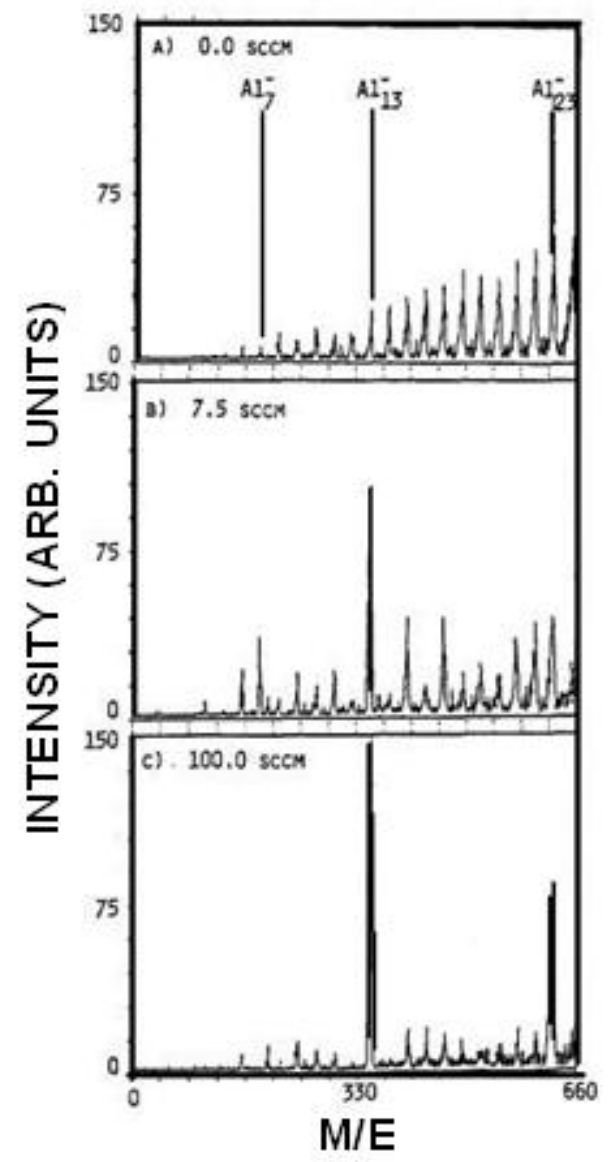

Fig. 7. Series of mass spectra showing progression of etching reaction of aluminum anions $\left(\mathrm{Al}_{5}{ }^{-}-\mathrm{Al}_{24}{ }^{-}\right)$ with oxygen. Note that these are all on the same intensity scale clearly showing production of $\mathrm{Al}_{13}{ }^{-}$and $\mathrm{Al}_{23}{ }^{-}:$(a) 0.0 sccm oxygen, (b) 7.5 sccm oxygen, (c) 100.0 sccm oxygen [Ref. 20] 


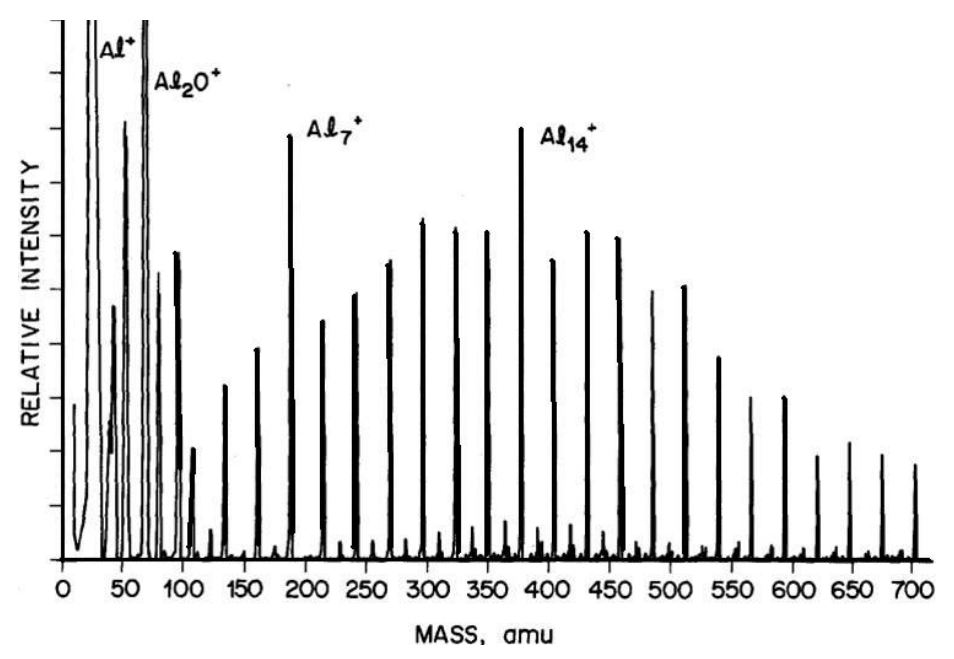

Fig. 8. Mass spectrum of aluminum cluster ions. [Ref. 21]

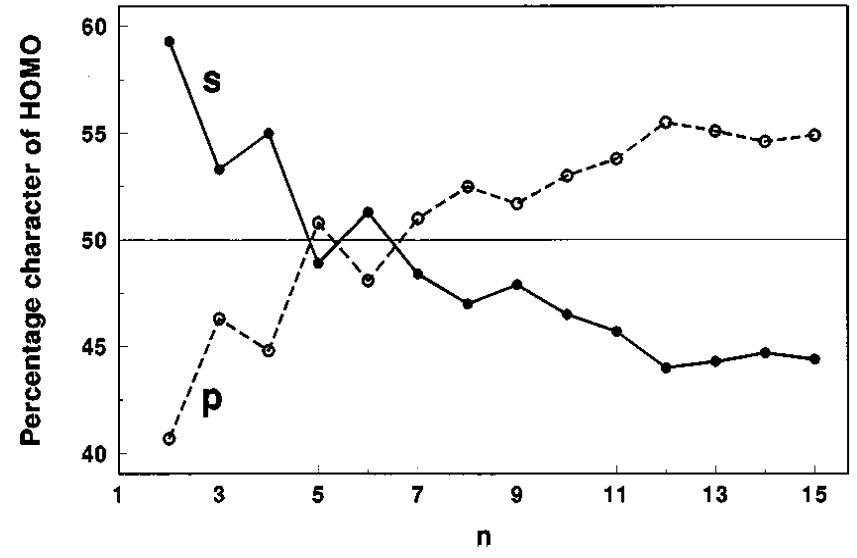

Fig. 9. Concentration of $\mathrm{s}$ and p-type electrons in the highest occupied molecular orbital (HOMO) of aluminum clusters. [Ref. 23]

The occurence of magic numbers can also be studied in the fragmentaion of clusters. It was predicted by Rao et al [24] that the preferred products in the fragmentation of a parent cluster should always contain a magic cluster. For example $\mathrm{Na}_{10}$ cluster would preferencially fragment into $\mathrm{Na}_{8}+\mathrm{Na}_{2}$. In the case of a charged cluster, for example $\mathrm{Na}_{10}{ }^{+}$, the preferred product would be $\mathrm{Na}_{9}{ }^{+}+\mathrm{Na}$. This is consistent with what is observed in nuclear fission and is another 
example where cluster physics and nuclear physics find common ground. Mass spectrometry experiments on the fragmentation of doubly charged $\mathrm{Na}_{n}{ }^{++}$clusters later performed by Brechignac et. al. [25] confirmed this asymmetric fission (see Fig. 10).

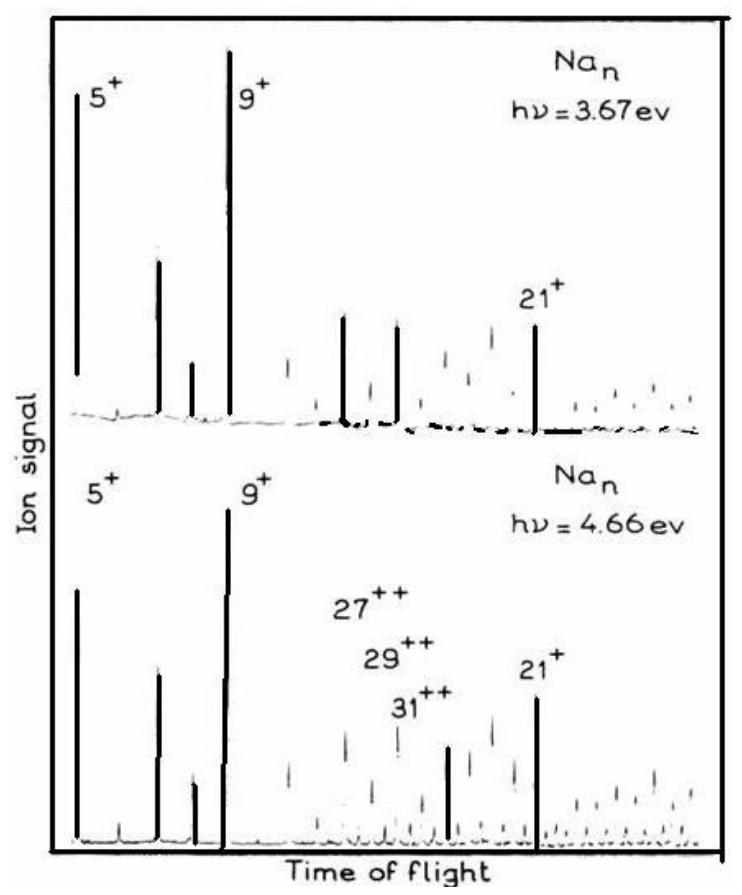

Fig.10. Mass spectra of photo ionized neutral-sodium-cluster distribution. Upper trace, obtained with photon energy of $3.67 \mathrm{eV}$ shows only singly charged clusters of $\mathrm{Na}_{n}{ }^{+}$. Lower trace, obtained at higher photon energy of $4.66 \mathrm{eV}$ puts into evidence doubly charged clusters $\mathrm{Na}_{n}{ }^{++}$from $n=27$. [Ref. 25]

\section{Clusters containing transition metal atoms - the 18-electron rule}

Transition metals with unfilled $d$-shells have different electronic structure than those of simple metals. Here the unfilled $d$-electrons are quasi-localized while the filled $s$-electrons are delocalized. Because of the quasi-localized $d$-electrons, transition metal atoms carry magnetic moments and their clusters can either be ordered ferromagnetically, antiferromagnetically, or even ferrimagnetically. Stability of clusters that involve transition metal atoms follow different electron counting rules than those described for simple metal clusters above. For example, no 
clusters of transition metal atoms containing 2, 8, 20, .. atoms are known to be magic species and the mass spectra do not show any well-defined set of prominent peaks. Instead, cluster stability is governed by the 18 -electron rule. Transition metal-based clusters stabilized by the 18electron rule can be metal-organic complexes such as $\mathrm{Cr}\left(\mathrm{C}_{6} \mathrm{H}_{6}\right)_{2}, \mathrm{Fe}\left(\mathrm{C}_{5} \mathrm{H}_{5}\right)_{2}$, and metal-carbonyls $\left[\mathrm{M}(\mathrm{CO})_{n}\right]$, inorganic complexes such as $\mathrm{WSi}_{12}$, or all-metal species such as $\mathrm{Au}_{12} \mathrm{~W}$.

Examples of these clusters are given in the following.

Transition metal carbonyls provide prototypical example of the 18-electron rule that predict the relative stabilities and coordination numbers of ligands in these complexes [26-29]. $\mathrm{Ni}(\mathrm{CO})_{4}, \mathrm{Fe}(\mathrm{CO})_{5}$, and $\mathrm{Cr}(\mathrm{CO})_{6}$ are well-known 18-electron neutral carbonyls. Recent gas phase work by Duncan and coworkers [27-29] has shown that their isoelectronic cations, $\mathrm{Cu}\left(\mathrm{CO}_{4}\right)^{+}$, $\mathrm{Co}(\mathrm{CO})_{5}{ }^{+}$, and $\mathrm{Mn}(\mathrm{CO})_{6}{ }^{+}$are also stable and have the same structures as the neutrals. Consistent with the 18-electron rule, the number of carbonyl ligands in the early transition metals such as Sc and $\mathrm{Y}$ has to increase to achieve stability. Using gas phase laser vaporization technique, infrared spectroscopy, and theoretical calculations Duncan and coworkers have studied the structure and stability of $\mathrm{Sc}(\mathrm{CO})_{n}{ }^{+}$and $\mathrm{Y}(\mathrm{CO})_{n}{ }^{+}$complexes [26]. The corresponding mass spectra are shown in Fig. 11. Note that the prominent peak at $\mathrm{Y}(\mathrm{CO})_{8}{ }^{+}$is consistent with the 18-electron rule. The enhanced stability of this cluster is also evident from the photo dissociation of $\mathrm{Y}(\mathrm{CO})_{9}{ }^{+}$where the dominant fragment corresponds to $\mathrm{Y}(\mathrm{CO})_{8}{ }^{+}$. This is consistent with the earlier prediction that the most prominent by-product in the fragmentation of a cluster is a magic cluster [24]. However, all transition metal carbonyls do not form the 18-electron complex. For example, $\mathrm{Sc}^{+}$ yields a 16-eletron seven-coordinate species $\mathrm{Sc}(\mathrm{CO})_{7}{ }^{+}$which has a $C_{3 v}$ capped octahedral structure while $\mathrm{Y}(\mathrm{CO})_{8}{ }^{+}$forms a $D_{4 d}$ square antiprism. 


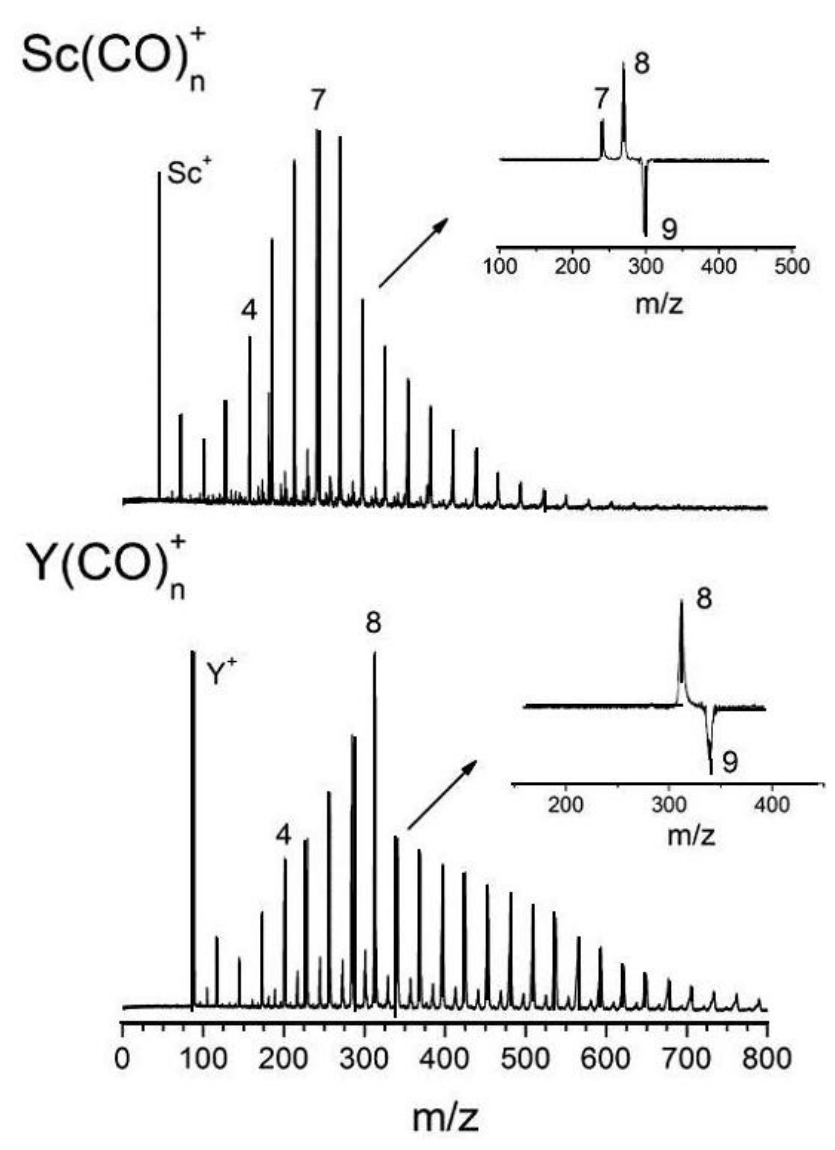

Fig. 11. Mass spectra of $\mathrm{M}(\mathrm{CO})_{n}{ }^{+}$ions $(\mathrm{M}=\mathrm{Sc}, \mathrm{Y})$ produced from laser vaporization source. Weaker mass peaks in between the carbonyls are oxides from sample impurities. The insets show representative photodissociation patterns for scandium and yttrium carbonyl cations. [Ref. 26]

The magic $\mathrm{WSi}_{12}$ cluster observed by Hiura et al [30] in the reaction of $\mathrm{W}^{+}$with $\mathrm{SiH}_{4}$ provides an example of an inorganic transition metal-based cluster that is stabilized by the 18electron rule. The sequential growth starting from $\mathrm{W}^{+}$to $\mathrm{WSi}_{12} \mathrm{H}_{x}^{+}$is shown in the time-resolved mass spectra in Fig. 12 [30]. The growth rate of $\mathrm{WSi}_{n} \mathrm{H}_{x}{ }^{+}$decreased with increasing $n$ and clusters with $n>12$ were rarely found in the longest holding time. This shows that $\mathrm{WSi}_{12} \mathrm{H}_{x}^{+}$ cluster is unreactive towards $\mathrm{SiH}_{4}$ for $n>12$. Assuming that each $\mathrm{Si}$ contributes one electron to the overall bonding of the cluster, $\mathrm{WSi}_{12}$ cluster would contain 18 -electrons, and hence is stable. This was also confirmed by theoretical calculation and the geometry of $\mathrm{WSi}_{12}$ is that of a $\mathrm{W}$ 
atom sandwiched between two hexagonal arrangements of $\mathrm{Si}_{6}$ moiety, similar to that of $\mathrm{Cr}\left(\mathrm{C}_{6} \mathrm{H}_{6}\right)_{2}$. The 18-electron rule was recently used to predict that an all-metal W@ $\mathrm{Au}_{12}$ complex can be a magic cluster [31] which was subsequently verified by experiment [32].

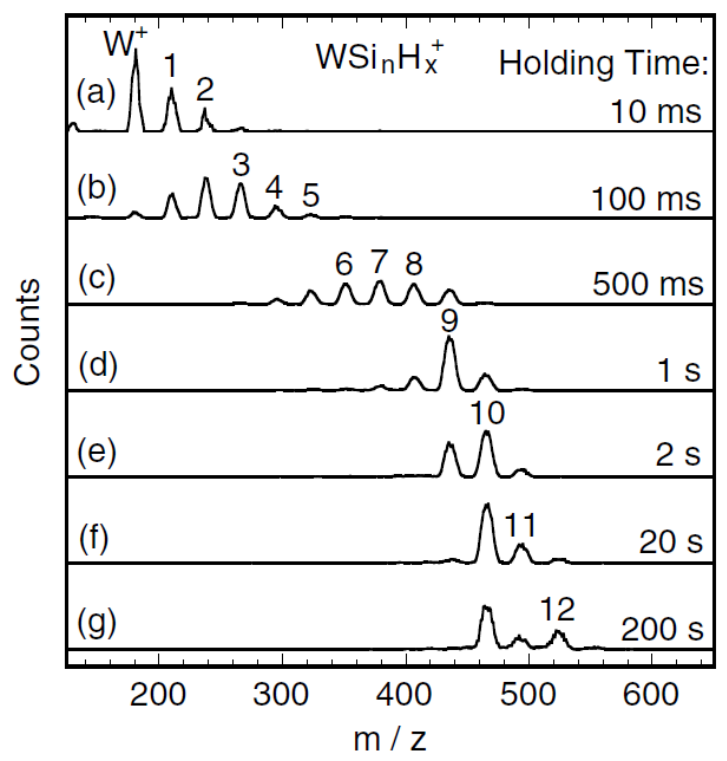

Fig.12. Time-resolved mass spectra of $\mathrm{WSi}_{n} \mathrm{H}_{x}^{+}$cluster ions produced in the ion trap. Each spectrum was observed after a given holding time: (a) $10 \mathrm{~ms}$, (b) $100 \mathrm{~ms}$, (c) $500 \mathrm{~ms}$, (d) $1 \mathrm{~s}$, (e) $2 \mathrm{~s}$, (f) $20 \mathrm{~s}$, and (g) $200 \mathrm{~s}$. The numbers above the peaks represent the number of $\mathrm{Si}$ atoms, $n$, in $\mathrm{WSi}_{n} \mathrm{H}_{x}^{+}$. [Ref. 30]

\section{Clusters of covalently bonded atoms}

(i) $\quad C$ and $S i$

Carbon and silicon are two of the most important elements in the periodic table, carbon forming the basis of life and silicon forming the basis of electronics. Both form covalent bonds, but their properties are very different, in crystals as well as in clusters. While carbon in the bulk phase forms $s p^{1}, s p^{2}$, and $s p^{3}$ bonds and exhibits stable structures in one, two, and three dimensions, silicon forms $s p^{3}$ bonds. In clusters, $\mathrm{C}$ exhibits the well-known fullerene cage 
structures while Si forms compact structures. The most spectacular impact of mass spectrometry in cluster science has been the observation of a single peak that corresponds to a cluster of $60 \mathrm{C}$ atoms. In Fig. 13 we reproduce the original spectra of carbon clusters taken under different experimental conditions [33]. Fig 13 (c) was obtained when the effective helium density over the graphite target was less than 10 torr and was believed to represent cluster distribution that simply resulted from pieces of the graphite sheet ejected in the primary vaporization process. Fig. 13 (b) was obtained when roughly 760 torr helium was present over the graphite target at the time of laser vaporization. Fig. 13 (a) was the result of further maximizing the cluster thermalization and cluster-cluster reaction conditions. The singularly prominent peak corresponding to $\mathrm{C}_{60}$ points to the unusual stability of this cluster. Smalley and coworkers [33] suggested a soccer-ball-like structure for this remarkably stable cluster. With 12 pentagons and 20 hexagons, this structure has 60 vertices, one for each $\mathrm{C}$ atom. This structure would have all valences satisfied by two single bonds and one double bond. Later synthesis of this cluster in bulk quantities by Kratschmer et al [34] allowed the experimental determination of its structure which matched exactly what Smalley and coworkers had envisioned. This discovery of a new form of carbon gave birth to new carbon science. Subsequent discovery of carbon nanotubes [35] and functionalized fullerenes has spunned technological potentials of the new nano-carbon phase. It is to be noted that mass spectrometry was at the root of this revolution in nano-carbon sceince. 


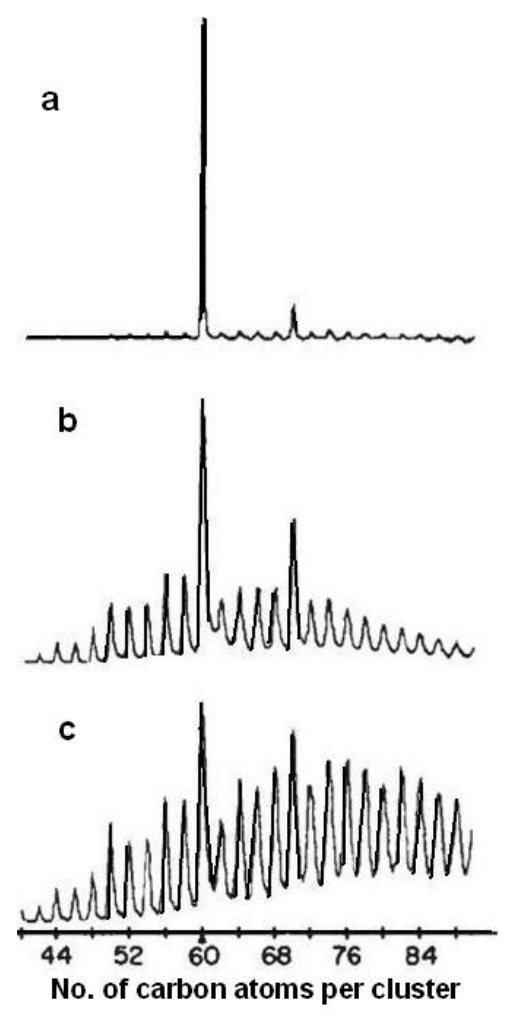

Fig. 13. Time-of-flight mass spectra of carbon clusters prepared by laser vaporization of graphite and cooled in a supersonic beam. The three spectra shown differ in the extent of helium collisons occuring in the supersonic nozzle. [Ref. 33]

Following the discovery of $\mathrm{C}_{60}$ considerable attention, both experimental and theoretical, was focussed on undersatnding the evolution of the carbon cluster geometries. For small clusters containg less than 10 atoms information on their structure were available from spectroscopic data and recativity patterns. The latter indicated unambiguously [36] the presence of different isomeric forms of $\mathrm{C}_{n}^{+}(\mathrm{n}=6,7,8)$ and the photoelectron studies [37] suggested a structural "phase" transition near $\mathrm{C}_{10}$. Using ion mobility experiments Bowers and coworkers [38] systematically studied the structure of carbon cluster ions from 3 to 60 atoms and demonstrated a transition from linear to ring to fullerene structures. The mobility of $\mathrm{C}_{n}{ }^{+}$clusters as a function of 
$n$ measuerd by these authors is shown in Fig. 14. It not only shows the sizes where strucrural transition takes place, but also illustrates the size ranges where different isomers are present.

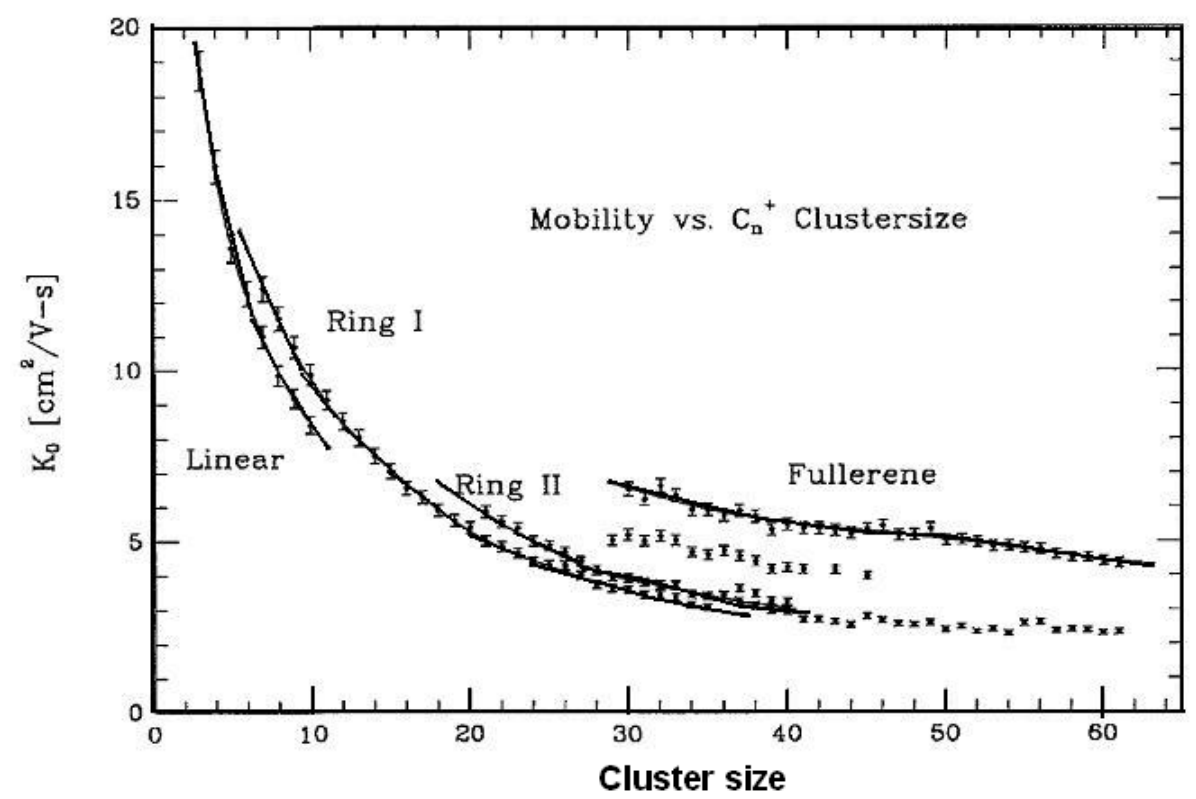

Fig. 14. A plot of the mobility vs cluster size for the positively charged cluster ions from $n=3$ to $n=60$. Note that all clusters from $n=29$ to 41 have at least three different mobilities corresponding to at least three resolved peaks in the arrival time distributions. The lines through the data points are merely to guide the eye for the four labeled families of isomers. At $n=37$ a new familiy starts. Only four points are shown $(n=37,38,39,40)$ but this family almost certainly continues above $n=40$. Unfortunately, the authors could not resolve the Ring II family and this new family (Ring III) above $n=40$. The unlabeled family from $n=29$ to $n=45$ is what the authors term "open" fullerene since it has a mobility intermediate between the planar ring systems and the fullerenes. While $\mathrm{C}_{3}{ }^{+}$and $\mathrm{C}_{4}{ }^{+}$are grouped in the linear family, the resolution is such that these clusters could either be linear or cyclic (or bent). [Ref. 38]

As pointed out earlier, although $\mathrm{Si}$ shares the same valence as $\mathrm{C}$, it does not behave like $\mathrm{C}$ either in crystals or in clusters. For example, all attempts to find pure $\mathrm{Si}_{60}$ in the fullerene structure have failed. Instead, Si clusters exhibit a different class of magic numbers. This was addressed experimentally by Bloomfield et al [39] in photofragmentation speactra of $\mathrm{Si}_{n}{ }^{+}$ 
clusters. In a typical experiment clusters are first ionized and then mass selected and their intensity determined. From the relative abunadance of the ionized clusters, information on the most stable clusters is obtained. Fragmentation of clusters can compete with ionization under many experimental conditions, making correspondence between strong cluster-ion signal and a "magic" neutral-cluster size uncertain. To avoid ambiguities associated with ionization, the above authors studied the properties of Si clusters after they were ionized and mass selected. In Fig. 15 we reproduce the mass spectra of $\mathrm{Si}_{n}{ }^{+}$ions resulting from the photofragmentation of $\mathrm{Si}_{12}{ }^{+}$ clusters [39]. The results show $\mathrm{Si}_{6}{ }^{+}$and $\mathrm{Si}_{10}{ }^{+}$to be unusually abundant ionic clusters. This is consistent with the thermodynamic stability derived from total energy calculations [40]. The structures of these magic clusters has since been verified by Raman spectra of size selected Si clusters [41] and more recently from direct visualization of reversible dynamics of $\mathrm{Si}_{6}$ cluster embedded in a graphene pore [42].

Structures of metal-silicon cluster cations, $\mathrm{MSi}_{n}{ }^{+}(\mathrm{M}=\mathrm{Cu}, \mathrm{Ag}, \mathrm{Cr})$ as well as the unusual stability of $\mathrm{Si}_{10}{ }^{+}$cluster have also been inferred from photodissociation studies [43]. In Fig. 16 we show the photodissociation spectra of mass-selected $\mathrm{AgSi}_{7}{ }^{+}$and $\mathrm{AgSi}_{10}{ }^{+}$cluster ions. Photodissociation of $\mathrm{AgSi}_{10}{ }^{+}$yields $\mathrm{Si}_{10}{ }^{+}$indicating that it is a magic number. In addition, the loss of a metal atom during photodissociation also indicates that the metal atom sits on the surface of the Si cluster. Photofragmentation of $\mathrm{CrSi}_{15}{ }^{+}$, on the other hand, tells a different story [Fig. 17]. We see from Fig. 17 the presence of $\mathrm{CrSi}_{13}{ }^{+}$and $\mathrm{CrSi}_{14}{ }^{+}$indicating that at these sizes $\mathrm{Cr}$ atom may be occupying an endohedral position. 


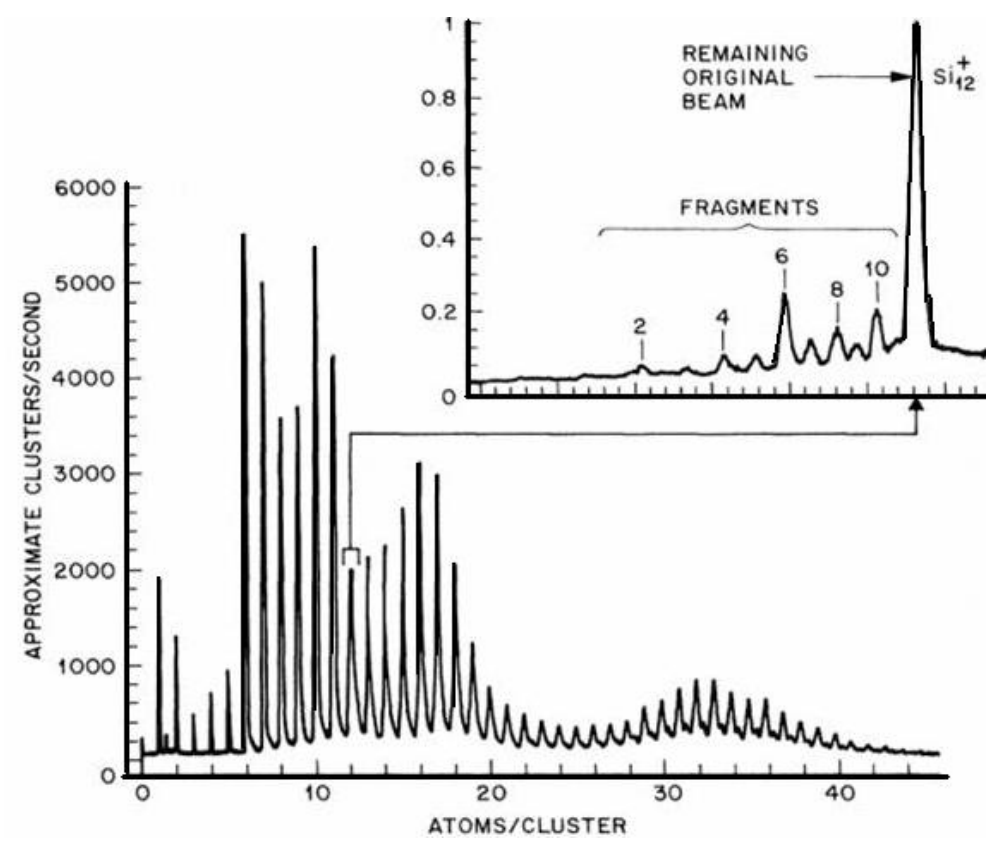

Fig. 15. A spectrum of small to medium Si cluster ions. An example of the preselection of cluster size is shown on the upper right where a single ion mass, $\mathrm{Si}_{12}{ }^{+}$, is isolated and fragmented to produce the fragmentation pattern. [Ref. 39]

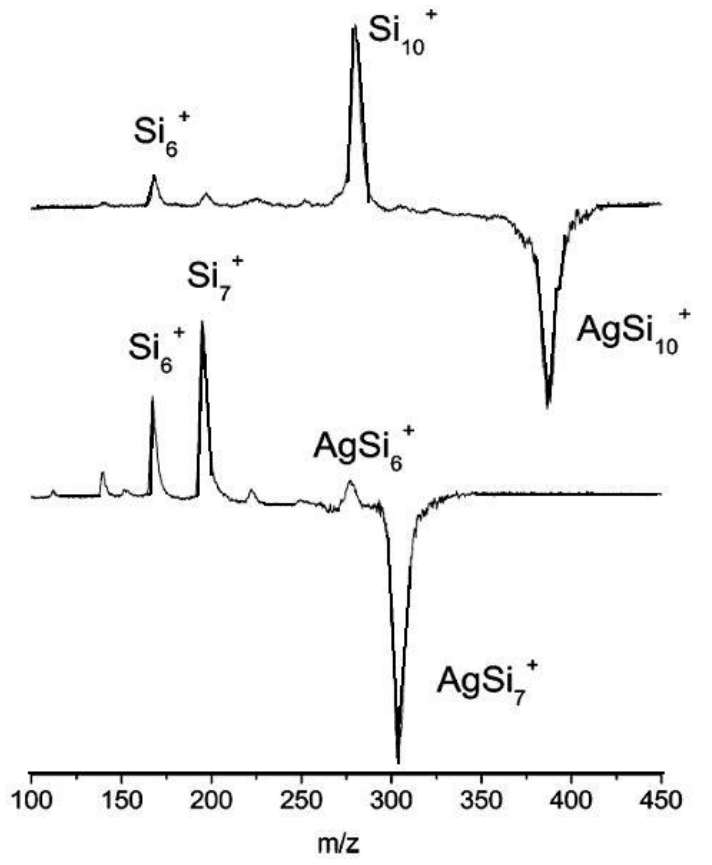


Fig. 16. Photodissociation of mass-selected $\mathrm{AgSi}_{7}{ }^{+}$and $\mathrm{AgSi}_{10}{ }^{+}$at 532 nm. [Ref. 43]

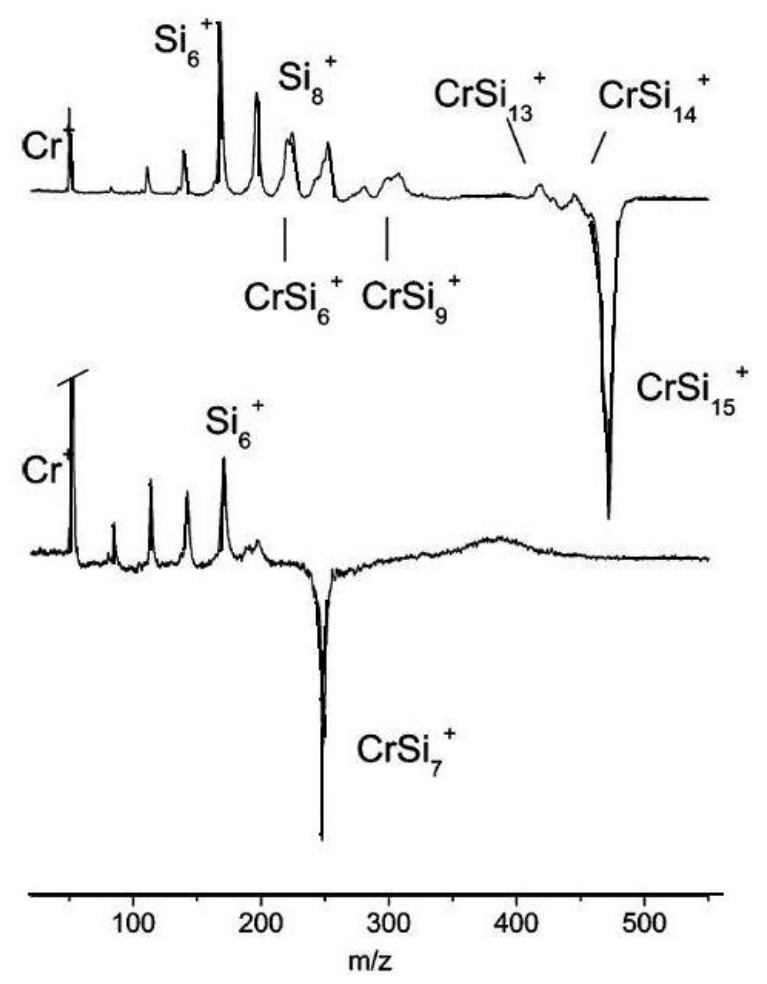

Fig. 17. Photodissociation of mass-selected $\mathrm{CrSi}_{7}{ }^{+}$and $\mathrm{CrSi}_{15}{ }^{+}$at $355 \mathrm{~nm}$. [Ref. 43]

(ii) Transition metal-oxide clusters

Mass spectrometry has also helped to elucidate the structure, stability, and reactivity of metal oxide clusters where significant covalency exists. As examples we consider transition metal-oxides which are widely used as both catalysts and catalytic supports. However, a molecular level understanding of their function has been difficult to achieve. The advantage of mass spectrometry is that it allows to mass-select a specific cluster whose chemical activity can be studied without the influence of its environment. Seminal studies by Castleman [44-46] and Duncan's [47-50] groups on oxide clusters of $\mathrm{V}, \mathrm{Fe}, \mathrm{Co}, \mathrm{Zr}, \mathrm{Nb}$, etc. have shown dramatic patterns with specific stoichiometries throughout the mass range and compositions not found in 
bulk solids. Photodissociation experiments by Duncan and coworkers have further shown that the abundant masses in the cluster growth are even more prominent in fragmentation channels, thus confirming the stability of these units. As examples we show in Fig. 18 mass distributions of $\mathrm{V}, \mathrm{Nb}$, and Ta oxide cluster ions [44]. The first prominent peak in $\mathrm{V}$-oxide cluster ions occurs at $\mathrm{VO}^{+}$while those for $\mathrm{Nb}$ and Ta-oxide cluster ions, the first peak arises at $\mathrm{MO}_{2}^{+}$. Similar differences exist in the stoichiometriess of other prominent peaks. Collision-induced photodissociation experiments can further establish the relative stability of mass-selected metaloxide cluster ions. For example, in the V-oxide group of cluster cations $\mathrm{MO}_{2}{ }^{+}$and $\mathrm{M}_{3} \mathrm{O}_{7}{ }^{+}$are favored while $\mathrm{MO}_{2}, \mathrm{MO}_{3}$, and $\mathrm{M}_{2} \mathrm{O}_{5}$ are the favored neutral products. Photodissociation experiments of $\mathrm{V}, \mathrm{Nb}$, and Ta oxide cluster cations by Duncan and coworkers [50] have led to similar results. These authors observed that dissociation occurs by either eliminating $\mathrm{O}$ or by fission. In $\mathrm{V}$-system the cations tend to loose $\mathrm{O}_{2}$ while $\mathrm{Nb}$ - and Ta-species loose $\mathrm{O}$. For each metal increment, oxygen elimination proceeds until a terminal stoichiometry is reached. Clusters having terminal stoichiometry no longer eliminate $\mathrm{O}$, but rather undergo fission. Similar results have been obtained for Fe-, Cr-, and Zr-based systems [47, 48]. 


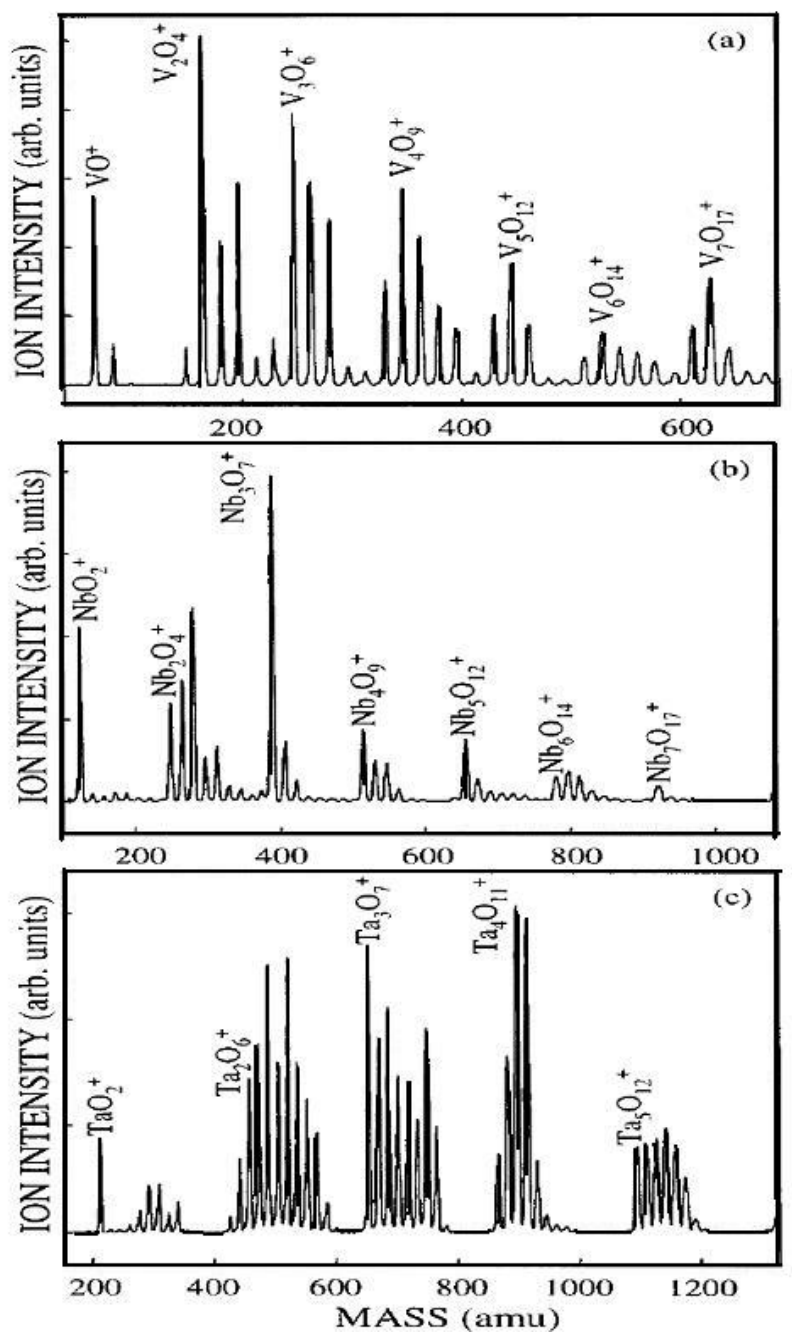

Fig. 18. Mass distributions of (a) vanadium, (b) niobium, and (c) tantalum oxide cluster cations. [Ref. 44]

Contrary to the conventional understanding that the structure of atomic clusters usually differ from the bulk constituents of which they are comprised, Sun et al [51] showed that even a dimer of tungsten oxide $\left(\mathrm{WO}_{3}\right)_{2}$ possesses bulk-like features and geometry of a small cluster containing only 4 tungsten and 12 oxygen atoms bears the hallmarks of crystalline tungsten oxide, $\mathrm{WO}_{3}$. This observation was validated by a synergistic approach involving mass distributions under quasi-steady state conditions and first principles molecular orbital theory. In 
Fig. 19 we show the mass ion spectra of $\mathrm{WO}_{3}$ clusters [51]. It is seen that all the major peaks correspond to clusters whose building blocks are $\mathrm{WO}_{3}$, the same stoichiometry as in the bulk. Comparison of the computed electron affinity with experimental photoemission spectra further confirmed the accuracy of the calculated geometries. A recent experiment by Bowen and coworkers [52] has confirmed the existence of these "baby" crystals in PbS clusters containing as few as 32 monomers. These studies illustrate that in strongly covalent materials even tiny clusters can act as the embryonic form of their crystalline phase. This lends the possibility that a fundamental understanding of complex processes such as catalytic reactions on surfaces may be achieved on an atomic scale with clusters as model systems.

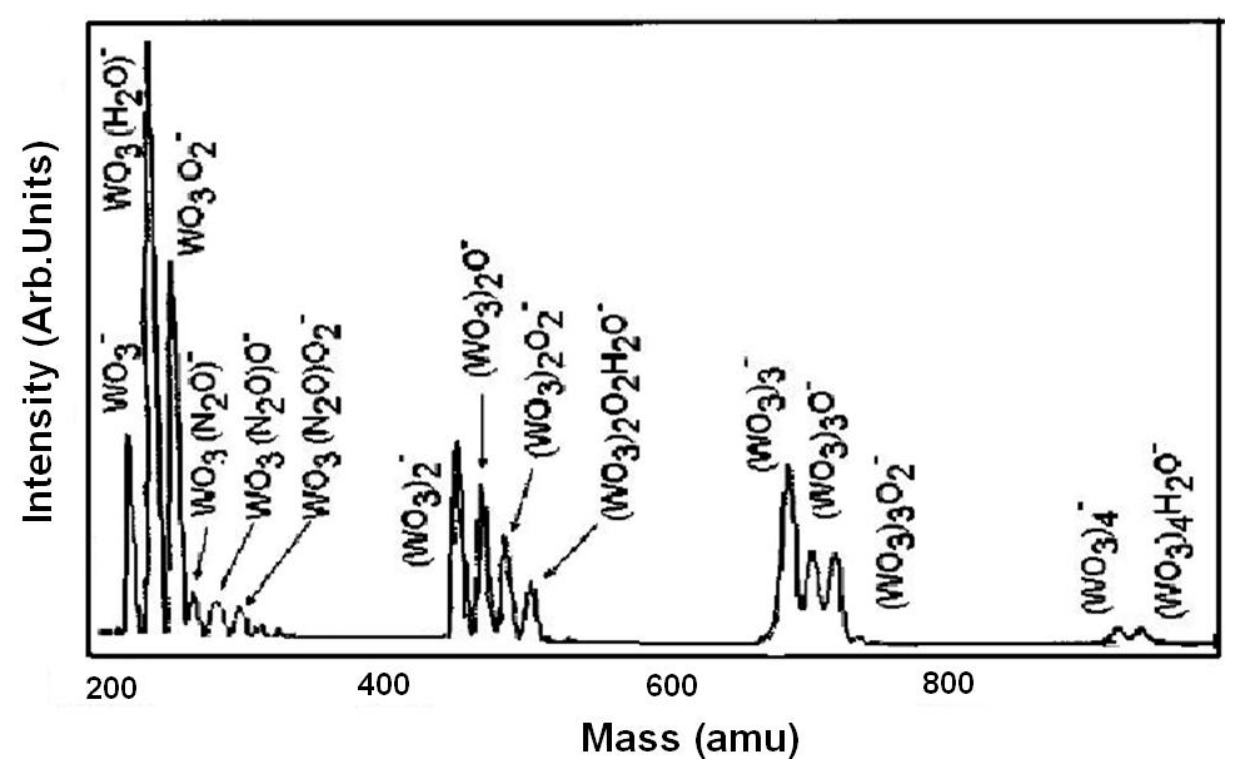

Fig. 19. Mass spectra of $\mathrm{WO}_{3}$ clusters [Ref. 51]

Mass spectrometry has also enabled an understanding of the reactivity patterns of sizespecific transition metal clusters [53-56]. The results show remarkable sensitivity of cluster sizes to reactions and certain cluster size react 10-100 times more efficiently than others differing by only one atom in size. For example, a systematic study of $\mathrm{CO}$ chemisorption on transition metal 
(V, Fe, Co, Ni, Cu, Nb, Mo, Ru, Pd, W, Ir, and Pt) clusters containing up to 14 atoms by Cox et al [54] showed that CO is readily chemisorbed on most transition metal clusters containing five or more metal atoms and that the reactivity of larger clusters varied by a factor of 2 or 3 . Similarly, the reactivity of hydrogen to Fe clusters [53] was found to be a strong function of size and stable $\mathrm{Fe}_{x} \mathrm{H}_{y}$ clusters exist even though Fe does not form a hydride in the bulk phase.

Studies such as these show the synergistic role of cluster science in furthering an understanding of the fundamentals of catalysis that impinges on virtually every field from energy production and usage to manufacturing and health.

\section{E: Compound clusters - Met Cars}

Another class of clusters that has attracted considerable attention and where mass spectrometry played a central role is metallo-carbohedrene or "Met- Car" [57, 58]. These clusters comprised of 8 transition metal atoms bound to 12 carbon atoms were discovered by Castleman and coworkers [57] during the course of studying reactions of hydrocarbons with transition metals. The first member of the class to be identified had Ti as the metal component. In Fig. 20 we reproduce the mass distribution of $\mathrm{Ti}_{m} \mathrm{C}_{n}{ }^{+}$clusters generated from reactions of titanium with $\mathrm{CH}_{4}$ and $\mathrm{C}_{2} \mathrm{H}_{2}$. Note the "super magic" peak corresponding to $\mathrm{Ti}_{18} \mathrm{C}_{12}{ }^{+}$. Subsequently, clusters identified containing $\mathrm{Zr}$, Hf, $\mathrm{V}, \mathrm{Nb}, \mathrm{Mo}$, and $\mathrm{Cr}$ were also discovered in experiments [59] where metal atoms were introduced into the gas phase using a laser vaporization source and a quadruple mass spectrometer for product detection. A few species containing binary and a couple even with ternary metal components were found.

Considering the masses of the Met-Car constituents as well as well as isotopes in the parent components, it is evident that assigning the exact stoichiometric content is often a non- 
trivial task. However, this is made easier using established isotope substitution methods borrowed from the field of mass spectrometry. There are a number of examples in cluster science where this technique plays an important role. Most useful have been cases where masses of isotopes comprised of carbon-13 and deuterated hydrogen remain unchanged in one series of experiments, while displaying a specific mass shift in another.

Identifying the structure of met-cars has not been easy not only because of the existence of many isomers lying closely in energy, but also because of the difficulty associated with approximations in theoretical methods that limit the accuracy of the calculated energies. Initially it was proposed [57] that $\mathrm{Ti}_{8} \mathrm{C}_{12}$ has pentagonal dodecahedron geometry with $T_{h}$ point group. Ion chromatography experiments of Bowers and coworkers $[60,61]$ provided early evidence of the dodecahedral-type cage structures. The cage-like structure of met-cars is a sharp departure from the cubic structures that characterize the bulk transition metal carbides. Later theoretical studies predicted that $\mathrm{Ti}_{8} \mathrm{C}_{12}$ with $T_{h}$ symmetry will undergo Jahn-Teller distortion [62-64]. Lower symmetry structures with much lower energies have been proposed [65-70] and the most stable one is a tetrahedral cluster with $T_{d}$ symmetry [70]. The electronic structure of the $T_{d} \mathrm{Ti}_{8} \mathrm{C}_{12}$ is consistent with several experimental observations, such as low ionization potential and the photoelectron spectra including low electron affinity [71, 72]. Larger metal-carbon species studied by Duncan and coworkers [73, 74] and by A. W. Castleman, Jr. and group [58] in massselected laser photodissociation measurements showed stoichiometries characteristic of cubic structures. These species, $\mathrm{Ti}_{14} \mathrm{C}_{13}{ }^{+}$and $\mathrm{V}_{14} \mathrm{C}_{13}{ }^{+}$, were found to be especially abundant and believed to have stabilities comparable to those of the $\mathrm{M}_{8} \mathrm{C}_{12}$ species as photodissociation of larger metal-carbon clusters produced both $8 / 12$ and $14 / 13$ stoichiometries as abundant photofragments. 
Of the many unique properties displayed by Met-Cars, ones related to ionization characteristics have attracted the most interest. For example, the ionization potential of the Ti containing material was found to have an ionization potential less than $4.4 \mathrm{eV}$, with a progressively diminishing value below $4 \mathrm{eV}$ for $\mathrm{Zr}$-substituted-based material [75]. Considering the low ionization potential and the high value for the vibrational density of states of a Met-Car, a dominant role of energy relaxation via thermionic emission is both predicted and expected. Indeed, Met-Cars display significant delayed ionization upon photoexicitation.
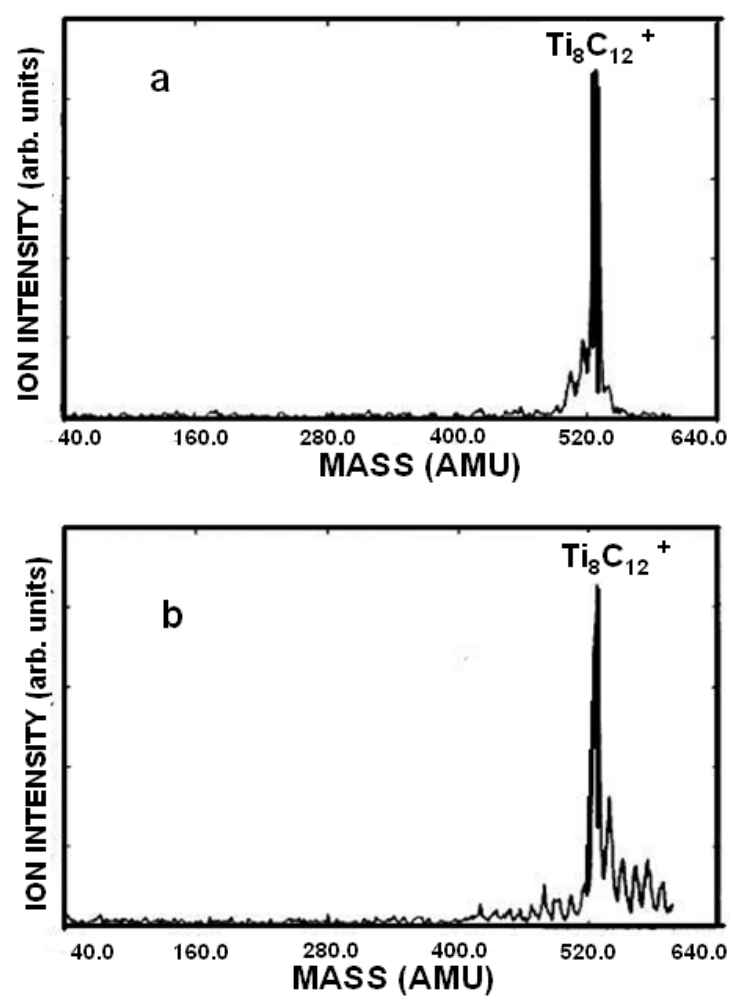

Fig. 20. (a) Mass distribution of $\mathrm{Ti}_{m} \mathrm{C}_{n}{ }^{+}$clusters generated from reactions of titanium with $\mathrm{CH}_{4}$. Note the "super magic" peak corresponding to $\mathrm{Ti}_{18} \mathrm{C}_{12}{ }^{+}$. (b) Mass distribution of $\mathrm{Ti}_{m} \mathrm{C}_{n}{ }^{+}$clusters generated from reactions of titanium with $\mathrm{C}_{2} \mathrm{H}_{2}$. Note the "super magic" peak corresponding to $\mathrm{Ti}_{18} \mathrm{C}_{12}{ }^{+}$. [Ref. 57]

\section{F: Clusters of ionically bonded atoms - TiN}


How small a cluster has to be before it can mimic the structure of its crystalline phase is one of the most fundamental questions in clusters science. For clusters that are characterized by metallic bonding, this is not an easy question to answer as the growth pattern does not show a clear trend. As mentioned above, in some oxide materials such as $\mathrm{WO}_{3}$, even very small clusters bear the hall mark of crystalline structure. However, examples where this can be seen clearly are in ionically bonded system such as TiN. It is readily seen that in many of these cases there is a close interplay between the fundamental structural arrangement of a building block and a discernible growth pattern from the element to a subset of a crystal. Several examples have been considered that deal with alkali halides, metal carbides, nitrides, and oxides. In Fig. 21 (a) we illustrate the evolution of crystal structure by considering the growth pattern of $(\mathrm{TiN})_{n}{ }^{+}$clusters [76]. From the abundance patterns in the time of flight mass spectrum one can easily see that the pronounced peaks correspond to cubic structures resembling pieces of the fcc lattice of solid TiN. In Fig. 21 (b) proposed structures of $(\mathrm{TiN})_{n}{ }^{+}$clusters based on magic numbers observed in the mass spectrum are given. 


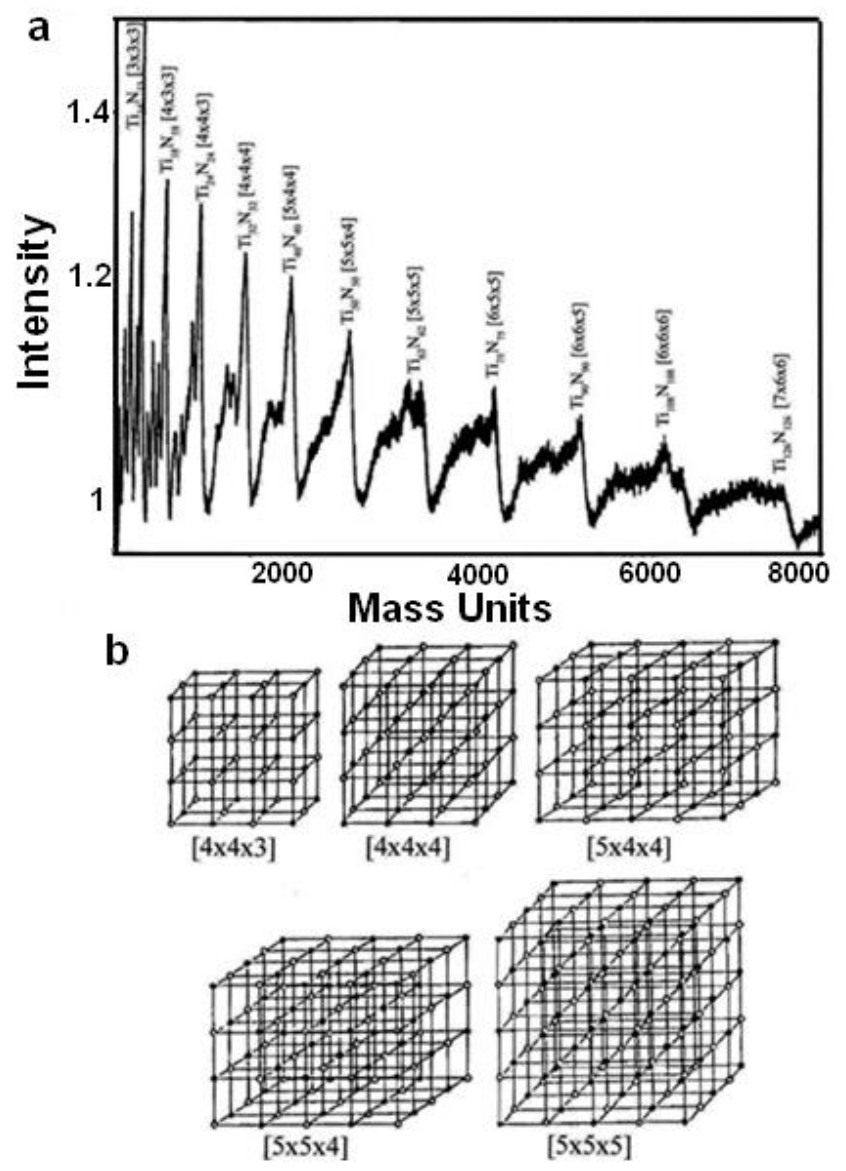

Fig. 21. Growth patterns of $(\mathrm{TiN})_{n}{ }^{+}$. (a) $\mathrm{TOF}$ mass spectrum of $(\mathrm{TiN})_{n}{ }^{+}$clusters. Abundance patterns indicate the clusters have cubic structures resembling pieces of the fcc lattice of solid TiN. (b) Proposed structures of $(\mathrm{TiN})_{n}{ }^{+}$clusters based on magic numbers observed in the mass spectrum. [Ref. 76]

\section{G: Clusters with electron deficient bonding - Wade-Mingos Rule}

\section{(i) Boranes and Alanes}

Boron is the lightest element that can form strong covalent bonds with hydrogen and boranes, a class of commonly known boron-hydrogen clusters, are well known for their unique structure and stability. For example, $\mathrm{B}_{12} \mathrm{H}_{12}{ }^{2-}$ is a very stable cage cluster where the $12 \mathrm{~B}$ atoms occupy the vertices of an icosahedron and the hydrogen atoms are radially bonded to each of the 
B atoms. The stability of this cluster in the di-anionic form is governed by the Wade-Mingos rule [77-79] which states that $(n+1)$ pairs of electrons are necessary to stabilize a borane polyhedron, where $n$ stands for the number of vertices. In such a closo-borane cluster two electrons are occupied in a covalent bond between $\mathrm{B}$ and $\mathrm{H}$ while the other two electrons are contributed to the cage bonding. The 26 electrons in $\mathrm{B}_{12} \mathrm{H}_{12}{ }^{2-}$ available for cage bonding, thus, satisfy the Wade-Mingos rule. Although Al and B belong to the same group in the periodic table, their chemistry is very different as we have seen to be the case with $\mathrm{C}$ and $\mathrm{Si}$. It has been a continuing puzzle why small Al-H clusters do not assume forms similar to those of boranes. A recent experiment by Bowen and coworkers [80] showed that some Al-H clusters do indeed mimic the structure and stability of boranes. In a study of the mass spectroscopy of $\mathrm{Al}_{x} \mathrm{H}_{y}$ clusters these authors found an intense peak corresponding to $\mathrm{Al}_{4} \mathrm{H}_{6}$. Analysis of the photoelectron spectrum of this cluster revealed a large HOMO-LUMO gap of $1.9 \mathrm{eV}$ which is characteristic of a stable species. Their data are reproduced in Fig. 22. The origin of the stability of $\mathrm{Al}_{4} \mathrm{H}_{6}$ was established through complimentary theoretical calculation. In Fig. 23 we show the equilibrium structure of $\mathrm{Al}_{4} \mathrm{H}_{6}$. It is composed of a tetrahedron whose vertices are occupied by 4 $\mathrm{Al}$ atoms. Four hydrogen atoms are radially bonded while the other two are bridge bonded on the opposite sides of the tetrahedron. The total number of electrons in this structure is 10 of which 8 electrons are contributed by the four $\mathrm{AlH}$ pairs and 2 are contributed by the bridge bonded $\mathrm{H}$ atoms. This is consistent with the Wade-Mingos rule and confirms that small Al-H clusters with specific size and composition can mimic the structure and stability of boranes. This has opened a new chapter in alane chemistry [81] which was possible because of the unique contribution of mass spectrometry. 

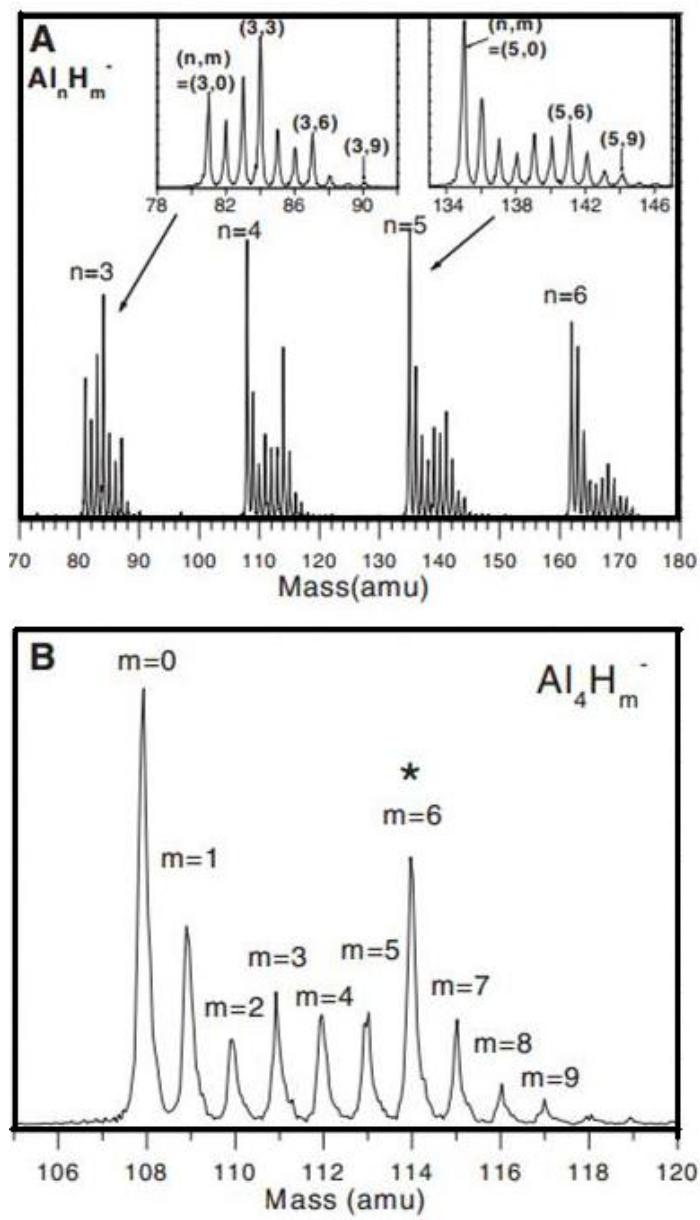

Fig. 22. (A) Mass spectrum showing the wide variety of $\mathrm{Al}_{n} \mathrm{H}_{m}{ }^{-}$anions that are formed with the PACIS source. Insets show magnified views of selected portions of the mass spectrum, revealing individual $\mathrm{Al}_{n} \mathrm{H}_{m}{ }^{-}$species. (B) A portion of the mass spectrum showing only $\mathrm{Al}_{4} \mathrm{H}_{m}{ }^{-}$series. [Ref. 80]

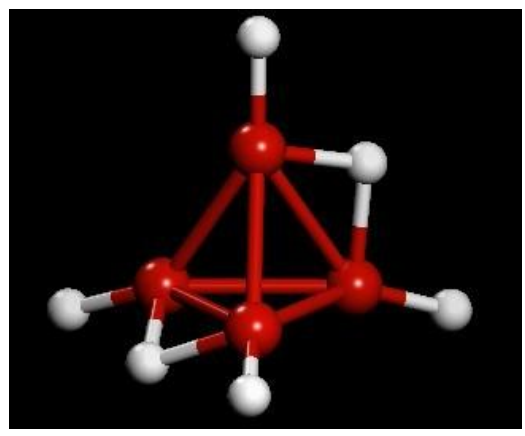

Fig. 23. The calculated structure of neutral $\mathrm{Al}_{4} \mathrm{H}_{6}$, shown to be a distorted tetrahedron [80] 
(ii) Zintl clusters

Zintl ions are multiply negatively charged polyatomic aggregates of main group elements and are often associated with the solution-based chemistry of naked clusters such as $\mathrm{Sn}_{5}{ }^{2-}$. While most studies of the Zintl anions are conducted in the condensed phases, there is a growing body of work in the gas phase where Zintl anions occur within larger clusters. The net charge on these anions is determined by the number of cationic moieties present in the system. Zintl ions involve bimetallic elements in Group IVA $(\mathrm{Sn}, \mathrm{Pb})$ and Group VA ( $\mathrm{Sb}, \mathrm{Bi})$. Group IVA elements contribute two electrons per atom making them electron deficient, and exist as anions in solution $\left(\right.$ eg. $\left.\mathrm{Sn}_{5}{ }^{2-}\right)$. Group VA elements, on the other hand, contribute three electrons per atom making them electron rich, and exist as cations (eg. $\mathrm{Bi}_{9}{ }^{5+}$ ). In Fig. 24 we show the mass distribution of $\mathrm{Pb}_{\mathrm{x}} \mathrm{Sb}_{\mathrm{y}}$ and isolelectronic $\mathrm{Sn}_{\mathrm{x}} \mathrm{Bi}_{\mathrm{y}}$ cluster cations of Duncan and coworkers [82] who were first to study Zintl ions in the gas phase. The magic numbers observed for $\mathrm{Pb}_{2} \mathrm{Sb}_{3}{ }^{+}$and $\mathrm{Sn}_{2} \mathrm{Bi}_{3}{ }^{+}$are consistent with $(n+1)$ pair valence electrons characteristic of closo- boranes. 


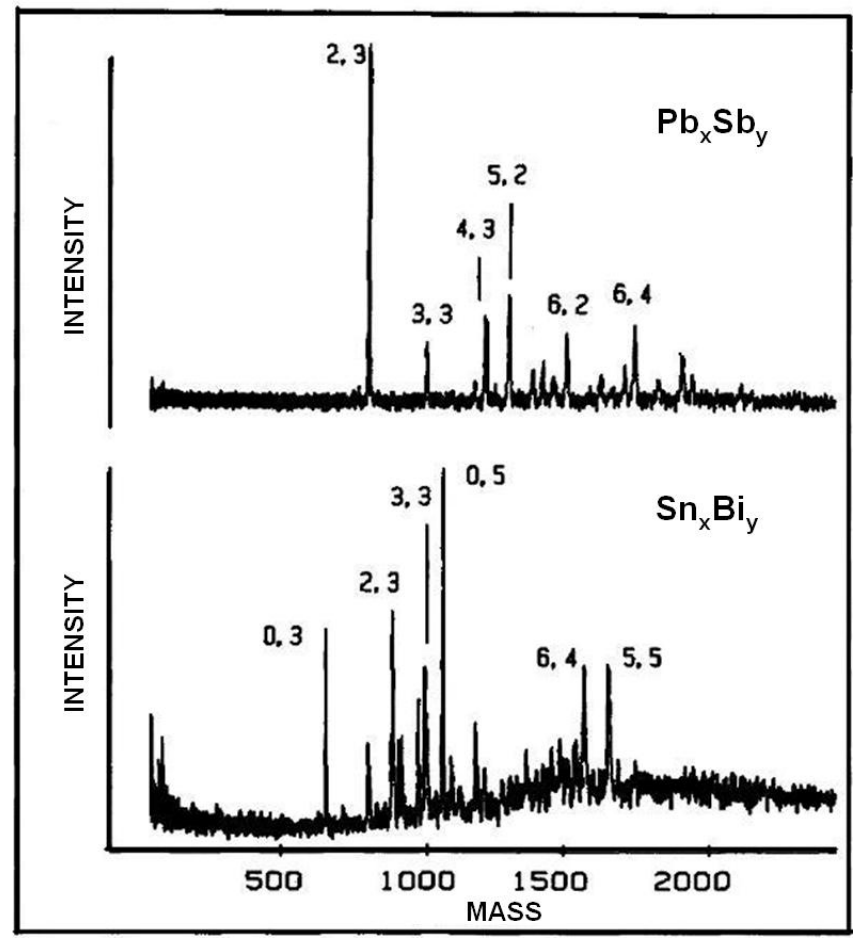

Fig. 24. Low power $\mathrm{ArF}$ photoionization $\left(0.05 \mathrm{~mJ} / \mathrm{cm}^{2}\right)$ of $\mathrm{Pb}_{\mathrm{x}} \mathrm{Sb}_{\mathrm{y}}$ and $\mathrm{Sn}_{\mathrm{x}} \mathrm{Bi}_{\mathrm{y}}$ cluster ion distributions [Ref. 82].

Further experimental evidence of the existence of Zintl ions in the gas phase came from the work of Farley and Castleman [83]. In Fig. 25 we show the mass spectra of $\mathrm{Bi}_{x} \mathrm{Na}_{y}{ }^{+}$clusters. Note that the peaks correspond to $(x, y)=(1,0),(2,3),(3,4),(4,5),(5,5),(6,5),(7,4),(8,5),(9,6)$ and $(14,7)$. Here cationic clusters $\mathrm{Bi}_{x} \mathrm{Na}_{y+1}{ }^{+}$, corresponding to $\mathrm{Bi}_{x}{ }^{y-}$, appear as magic numbers in the distribution for every reported anionic Zintl ion of bismuth. From the observation of $\mathrm{Bi}_{3} \mathrm{Na}_{4}{ }^{+}$as a magic number in the mass spectrum in Fig. 25 it can be inferred that $\mathrm{Bi}_{3}{ }^{3-}$, for example, is a Zintl anion. 


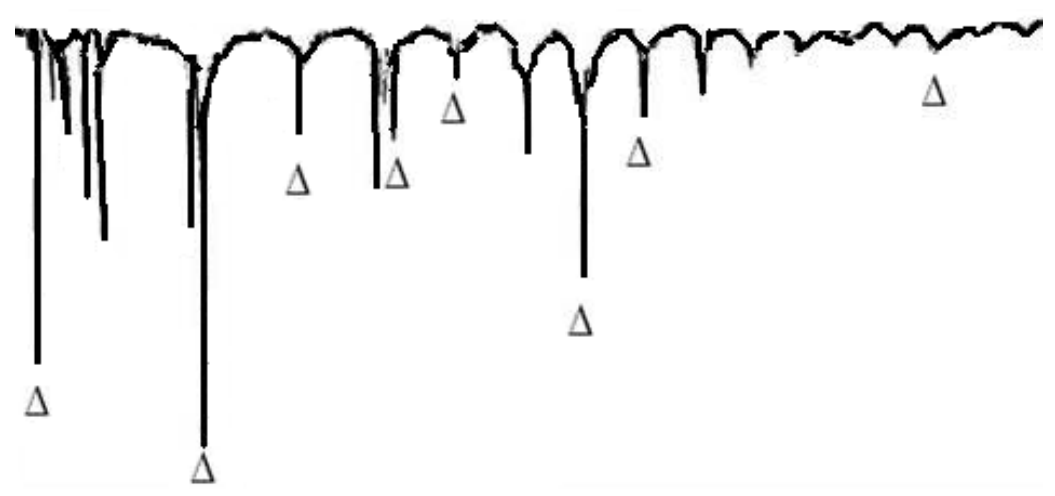

Fig. 25. $\mathrm{Bi}_{x} \mathrm{Na}_{y}{ }^{+}$cluster distribution [Ref. 83]

Many other Zintl anions such as $\mathrm{Sn}_{4}{ }^{4-}, \mathrm{Ga}_{4}{ }^{2-}, \mathrm{Sn}_{12}{ }^{2-}$, and $\mathrm{Pb}_{12}{ }^{2-}$ within the cluster anions $\left(\mathrm{Na}_{3} \mathrm{Sn}_{4}\right)^{-}$ , $\left(\mathrm{NaGa}_{4}\right)^{-},\left(\mathrm{KSn}_{12}\right)^{-}$, and $\left(\mathrm{KPb}_{12}\right)^{-}$, respectively, as well as endohedral $\left[\mathrm{M} @ \mathrm{~Pb}_{12}\right]^{2-}$ and $\left[\mathrm{M} @ \mathrm{Sn}_{12}\right]^{2-}$ where $\mathrm{M}$ is a transition metal atom have been studied through a combination of anion photoelectron spectroscopy and theorertical calculations [84-90]. Recently, aluminum moieties within selected Na-Al clusters have been shown to exhibit Zintl characterisitics [90]. Thus, one can see that the original discovery of the Zintl ions in the gas phase [82] seen through mass spectroscopy has had considerable impact on cluster science as well.

\section{Summary and Future Directions}

The retrospective presented in this paper has focused largely on employing various approaches coupled with mass spectrometry to deduce the electronic structure and stability of clusters. Although not solely on the subject of hard matter, much of the attention has gone to a discussion of metals, oxides, carbides and alloys. A subject that we briefly discussed but to which mass spectrometry has and continues to make a large contribution is the solvation phenomenon. Specifically, using a wide variety of available ion sources and coupling one of 
them to a suitable mass spectrometer, a search is undertaken to sample a gas phase distribution of clusters onto which is imposed a steady state distribution, over a wide range of temperatures. Appropriate analysis of the temperature dependent cluster distributions enables thermochemical data associated with solvation to be determined employing well known equations $\mathrm{I}(\mathrm{A})_{n}+\mathrm{A}=$ $\mathrm{I}(\mathrm{A})_{n+1}$. Here, the equation denotes intensity of $n$ and $n+1$ molecules of solvent A clustered to ion I, technically in units of chemical activity. This method depends on acquiring an equilibrium distribution for ligands A clustered to ion I. Under well-established conditions, a determination of the equilibrium constant with temperature, enthalpy and entropy data may be obtained. Subsequently, new insights into solvation are quantitatively determined.

It is difficult to imagine what was lying ahead in numerous areas of science and technology that was about to unfold as a consequence of the discovery of canal rays. Indeed, it is hard to find fields that have not been touched, ranging from energy production to medicine. Today, as we look forward we face a similar situation, whereupon potential advances are difficult to predict, but we see them arise so rapidly that we know new ones are waiting for identification. An area of research that is growing rapidly is "cluster science" which has impacts throughout nanoscience, as well numerous other areas. Of one thing we can be certain, Mass Spectrometry will play a major role in the future as it has in the past.

Acknowledgement: Research of PJ is supported in part by the U.S. Department of Energy, Office of Basic Energy Sciences, Division of Materials Sciences and Engineering under Award \# DE-FG02-96ER45579. We are thankful to Prof. Qian Sun for help with the figures. AWC gratefully acknowledges the Office of Naval Research N00014-12-1-0541 and this material is based upon work supported by the Air Force Office of Science Research under AFOR Award No. FA9550-10-1-0071 for financial support. 


\section{References:}

1. E. E. B. Campbell and M. Larsson, Eds. "Physics and Chemistry of Clusters-the Proceedings of the Nobel Symposium" (2001)

2. P. Jena and A. W. Castleman, Jr., Eds. "Nanoclusters- A Bridge Across Disciplines", Elsevier (2010)

3. J. A. Alonso, "Structure and Properties of Atomic Nanoclusters", Imperial College Press, (2005)

4. A. W. Castleman Jr and K. H. Bowen, Jr. Clusters, Structure, Energetics and Dynamics of Intermediate States of Matter, J. Phys. Chem. 1996, 100, 12911.

5. B. F. G. Johnson and J. C. McIndoe. Spectroscopic and mass spectrometric methods for the characterization of metal clusters. Coordination Chemistry Reviews. 2000, 200-202, 901.

6. T. G. Dietz, M. A. Duncan, D. E. Powers, and R. E. Smalley. Laser production of supersonic metal cluster beams J. Chem. Phys. 1981, 74, 6511

7. G. Gantefor, H. R. Siekmann, H. O. Lutz, and K. H. Meiswes-Broer, Pure metal and metaldoped rare-gas clusters grown in a pulsed ARC cluster ion source. Chem. Phys. Lett. 1990, 165,293

8. J. Q. Searcy and J. B. Fenn. Clustering of water on hydrated protons in a supersonic free jet expansion. J. Chem. Phys. 1974, 61, 5283.

9. X. Yang and A. W. Castleman, Jr. Large protonated water clusters $\mathrm{H}^{+}\left(\mathrm{H}_{2} \mathrm{O}\right)_{n}(1 \leq n \leq 60)$ : The production and reactivity of clathrate-like structures under thermal conditions. J. Am. Chem. Soc. 1989, 111, 6845.

10. J.-W. Shin, N. I. Hammer, E. G. Diken, M. A. Johnson. R. S. Walters, T. D. Jaeger, M. A. Duncan, R. A. Christie, and K. D. Jordan. Science, 2004, 304, 1137.

11. O. Echt, K. Sattler, and E. Recknagel. Magic Numbers for Sphere Packings: Experimental Verification in Free Xenon Clusters. Phys. Rev. Letters. 1981, 16, 1121.

12. K. Sattler, J. Muhlbach, O. Echt, P. Pfau, and E. Recknagel, Evidence for Coulomb Explosion of Doubly Charged Microclusters, Phys. Rev. Letters. 1981, 47, 160.

13. W. D. Knight, K. Clemenger, W. A. de Heer, W. A. Saunders, M. Y. Chou, and M. L. Cohen. Electronic Shell Structure and Abundances of Sodium Clusters. Phys. Rev. Lett. 1984, 52, 2141.

14. W. A. de Heer, K. Selby, V. Kresin, J. Masui, M. Vollmer, A. Chatelain, and W. D. Knight. Collective Dipole Oscillations in Small Sodium Clusters. Phys. Rev. Lett. 1987, 1805 (1987) 
15. G. F. Bertsch, P. F. Bortignon, and R. A. Broglia. Rev. Mod. Phys. 1983, 55, 287.

16. T.P. Martin, T. Bergmann, H. Gijhlich and T. Lange. Observation of electronic shells and shells of atoms in large Na clusters. Chem. Phys. Lett. 1990, 172, 209.

17. M. Manninen and P. Jena. Electronic Shell Structure and the crystal field splitting in simple metal clusters, Euro. Phys. Lett. 1991, 14, 643.

18. B. K. Rao, P. Jena, M. Manninen, and R. M. Nieminen. Spontaneous fragmentation of multiply charged metal clusters. Phys. Rev.Lett. 1987, 58, 1188.

19. N. D. Bhaskar, R. P. Frueholz, C. M. Klimcak, and R. A. Cook. Evidence of electronic shell structure in $\mathrm{Rb}^{+}(\mathrm{N}=1-100)$ produced in a liquid-metal ion source. Phys. Rev. B 1987, 36, 4418.

20. R. E. Leuchtner, A. C. Harms, and A. W. Castleman, Jr. Thermal metal cluster anion reactions: Behavior of aluminum clusters with oxygen, J. Chem. Phys. 1989, 91, 2753.

21. Martin F. Jarrold, J. Eric Bower, and J. S. Kraus. Collision induced dissociation of metal cluster ions: Bare aluminum clusters, $\mathrm{Al}^{+}{ }_{\mathrm{n}}(\mathrm{n}=3-26)$. J. Chem. Phys. 1987, 86, 3876.

22. X. Li, H. Wu, X. B. Wang, and L. S. Wang. $s-p$ Hybridization and Electron Shell Structures in Aluminum Clusters:A Photoelectron Spectroscopy Study Phys. Rev. Lett. 1998, 81, 1909.

23. B. K. Rao and P. Jena. Evolution of the electronic structure and properties of neutral and charged aluminum clusters: A comprehensive analysis. J. Chem. Phys.1999, 111, 1890.

24. P. Jena, B. K. Rao, and R. M. Nieminen. Fragmentation channels and their relationship to magic number studies of microclusters. Solid State Commun. 1986, 59, 509.

25. C. Brechignac, Ph. Cahuzac, F. Carlier, and M. de Frutos. Asymmetric Fission of $\mathrm{Na}_{n}{ }^{++}$ around the Critical Size of Stability. Phys. Rev. Lett. 1990, 64, 2893.

26. A. D. Brathwaite, J. A. Maner, and M. A. Duncan. Testing the limits of the 18-electron rule: The gas-phase carbonyls of $\mathrm{Sc}^{+}$and $\mathrm{Y}^{+}$. Inorg. Chem. 2014, 53, 1166.

27. A. D. Brathwaite, A. M. Ricks, and M. A. Duncan. Infrared photodissociation spectroscopy of Vanadium-carbonyls cations. J. Phys. Chem. A 2013, 117, 13435.

28. Z. D. Reed and M. A. Duncan. Infrared spectroscopy and structures of Manganese carbonyl cations, $\mathrm{Mn}(\mathrm{CO})_{n}{ }^{+}(n=1-9)$. Am. Soc. Mass. Spectroscopy. 2010, 21, 739.

29. A. M. Ricks, J. M. Bakker, G. E. Douberly, and M. A. Duncan. Infrared spectroscopy and structures of Cobalt carbonyl cations, $\operatorname{Co}(\mathrm{CO})_{n}{ }^{+}(n=1-9)$. J. Phys. Chem. A 2009, 113, 4701.

30. H. Hiura, T. Miyazaki, and T. Kanayama, Formation of metal-encapsulating Si cage clusters. Phys. Rev. Lett. 2001, 86, 1733. 
31. P. Pyykko and N. Runeberg. Icosahedral WAu 12 : A predicted closed-shell species, stabilized by aurophilic attraction and relativity and in accord with the 18-electron rule, Angew. Chem. Int. Ed. 2002, 41, 2174.

32. X. Li, B. Kiran, J. Li, H. Zhai, and L. S. Wang. Experimental observation and confirmation of icosahedral W@ $\mathrm{Au}_{12}$ and Mo@Au $\mathrm{Au}_{12}$ molecules. Angew. Chem. Int. Ed. 2002, 41, 4786.

33. H. W. Kroto, J. R. Heath, S. C. O'Brien, R. F. Curl, and R. E. Smalley. C 60 : Buckminsterfullerene. Nature, 1985, 318, 162.

34. W. Kratschmer, L. D. Lamb, K. Fostiropolous, and D. R. Huffman, Solid $\mathrm{C}_{60}$ : a new form of carbon. Nature, 1990, 347, 354.

35. S. Iijima. Helical microtubules of graphitic carbon, Nature, 1991, 354, 56.

36. D. C. Parent and S. W. McElvany. J. Am. Chem. Soc. 1989, 111, 2393.

37. S. H. Yang, C. L. Pettiette, J. Conceicao, O. Cheshnovsky, and R. E. Smalley. Ups of buckminsterfullerene and other large clusters of carbon. Chem. Phys. Lett. 1987, 139, 233.

38. G. von Helden, M.-T. Hsu, P. R. Kemper, and M. T. Bowers. Structures of carbon cluster ions from 3 to 60 atoms: Linears to rings to fullerenes. J. Chem. Phys. 1991, 95, 3835.

39. L. A. Bloomfield, R. R. Freeman, and W. L. Brown. Photofragmentation of Mass-resolved $\mathrm{Si}_{2-12}{ }^{+}$clusters. Phys. Rev. Lett. 1985, 54, 2246.

40. K. Raghavachari and C. M. Rohlfing. Bonding and stabilities of small silicon clusters: A theoretical study of $\mathrm{Si}_{7}-\mathrm{Si}_{10}$, J. Chem. Phys. 1989, 89,2219.

41. E. C. Honea, A. Ogura, C. A. Murray, K. Raghavachari, W. O. Sprenger, M. F. Jarrold, and W. L. Brown. Raman spectra of size-selected silicon clusters and comparison with calculated structures. Nature, 1993, 366, 42.

42. J. Lee, W. Zhou, S. J. Pennycook, J. Idrobo, and S.T. Pantelides. Direct visualization of reversible dynamics in a Si6 cluster embedded in a graphene pore. Nature Commun. 2013, 4, 1650.

43. J. B. Jaeger, T. D. Jaeger, and M. A. Duncan. Photodissociation of metal-silicon clusters: Encapsulated versus surface-bonded metal. J. Phys. Chem. A. 2006, 110, 9310.

44. K. A. Zemski, D. R. Justes, and A. W. Castleman, Jr. Studies of metal oxide clusters: Elucidating reactive sites responsible for the activity of transition metal oxide catalysts. J. Phys. Chem. B 2002, 106, 6136

45. N. A. Moore, R. Mitric, D. R. Justes, V. Bonacic-Koutecky, and A. W. Castleman, Jr. Kinetic analysis of the reaction between $\left(\mathrm{V}_{2} \mathrm{O}_{5}\right)_{n=1,2}{ }^{+}$and Ethylene, J. Phys. Chem. B 2006, 110, 3015 
46. R. C. Bell, K. A. Zemski, and A. W. Castleman, Jr. Size-specific reactivities of Vanadium oxide cluster cations. J. Cluster Sci. 1999, 10, 509

47. K. S. Molek, Z. D. Reed, A. M. Ricks, and M. A. Duncan. Photodissociation of Chromium oxide cluster cations. J. Phys. Chem. A 2007, 111, 8080

48. K. S. Molek, C. Anfuso-Cleary, and M. A. Duncan. Photodissociation of Iron oxide cluster cations. J. Phys. Chem. A 2008, 112, 9238

49. Z. D. Reed and M. A. Duncan. Photodissociation of Yttrium and Lanthanum oxide cluster cations. J. Phys. Chem. A 2008, 112, 5354

50. K. S. Molek, T. D. Jaeger, and M. A. Duncan. Photodissociation of vanadium, niobium, and tantalum oxide cluster cations. J. Chem. Phys. 2005, 123, 144313

51. Q. Sun, B. K. Rao, P. Jena, D. Stolcic, G. Gantefor, and A. W. Castleman, Jr. Appearance of bulk properties in small tungsten oxide clusters. J. Chem. Phys. 2004, 121, 9417.

52. B. Kiran, A. K. Kandalam, R. Rallabandi, P. Koirala, X. Li, X. Tang, Y. Wang, H. Fairbrother, G. Gantefoer, and K. Bowen. (PbS) $)_{32}$ : A baby crystal. J. Chem. Phys. 2012, 136, 024317.

53. R. L. Whetten, D. M. Cox, D. J. Trevor, and A. Kaldor. Correspondence between Electron Binding Energy and Chemisorption Reactivity of Iron Clusters. Phys. Rev. Lett. 1985, 54, 1494

54. D. M. Cox, K. C. Reichmann, D. J. Trevor, and A. Kaldor. CO chemisorption on free gas phase metal clusters. J. Chem. Phys. 1988, 88, 111

55. D. E. Bergeron and A. W. Castleman, Jr. Insights into the stability of silicon cluster ions: Reactive etching with $\mathrm{O}_{2}$. J. Chem. Phys. 2002, 117, 3219

56. E. K. Parks, L. Zhu, J. Ho, and S. J. Riley, The structure of small nickel clusters. I. $\mathrm{Ni}_{3}-\mathrm{Ni}_{15}$. J. Chem. Phys. 1994, 100, 7206

57. B. C. Guo, K. P. Kerns, and A. W. Castleman, Jr. $\mathrm{Ti}_{8} \mathrm{C}_{12}{ }^{+}$-Metallo-Carbohedrenes: A New Class of Molecular Clusters? Science. 1992, 255, 1411

58. S. Wei, B. C. Guo, J. Purnell, S. Buzza, and A. W. Castleman, Jr. Metallo-Carbohedrenes: Formation of Multicage Structures. Science 1992, 256, 818

59. J. S. Pilgrim and M. A. Duncan. Metallo-carbohedrenes: chromium, iron, and molybdenum analogs. J. Am. Chem. Soc. 1993, 115, 6958

60. G. von Helden, N. G. Gotts, P. Maitre, and M. T. Bowers. The structures of small ironcarbon cluster anions. Linear to planar to three-dimensional. Chem. Phys. Lett. 1994, 227, 601 
61. S. Lee, N. G. Gotts, G. von Helden, and M. T. Bowers. Evidence from Ion Chromatography Experiments That Met-Cars Are Hollow Cage Clusters. Science 1995, 267, 999.

62. A. Ceulemans and P. W. Fowler. Faraday communications. Bonding in $\mathrm{Ti}_{8} \mathrm{C}_{12}$ and the substitutional jahn-teller effect. J. Chem. Soc. Faraday Trans. 1992, 88, 2797

63. M. Rohmer, P. de Vaal, and M. Bernard. Jahn-Teller distortion predicted for metallocarbohedrenes: an ab initio SCF geometry optimization of the lowest singlet and triplet states of titanium carbide (Ti8C12) in the Th and D2h point groups. J. Am. Chem. Soc. 1992, 114,9696

64. H. Chen, M. Feyereisen, X. P. Long, and G. Fitzgerald. Phys. Rev. Lett. 1993, 71, 1732

65. I. J. Dance. Geometric and electronic structures of $\left[\mathrm{Ti}_{8} \mathrm{C}_{12}\right]$ : analogies with $\mathrm{C}_{60}$. J. Chem. Soc. Chem. Commun. 1992, 1779

66. M. Rohmer, M. Bernard, C. Henriet, C. Bo, and J. Poblet. $\mathrm{Ti}_{8} \mathrm{C}_{12}$ : a polytopal molecule with 36 Ti-C bonds. J. Chem. Soc. Chem. Commun. 1993, 1182

67. I. J. Dance. Nanocrystal [ $\mathrm{Ti}_{14} \mathrm{C}_{13}$ ] to Metallocarbohedrene $\left[\mathrm{Ti}_{8} \mathrm{C}_{13}\right]$ : Structural Principles and Mechanism. J. Am. Chem. Soc. 1996, 118, 2699

68. Z. Lin and M. B. Hall. Theoretical studies on the stability of $\mathrm{M}_{8} \mathrm{C}_{12}$ clusters. J. Am. Chem. Soc. 1993, 115, 11165

69. M. Rohmer, M. Bernard, C. Bo, and J. Poblet. Ab Initio SCF and CI Investigations on Titanium-Carbon Clusters: Metallocarbohedrenes $\mathrm{Ti}_{8} \mathrm{C}_{12}$ and $\mathrm{Cfc}$ Crystallites $\mathrm{Ti}_{14} \mathrm{C}_{13}$. J. Am. Chem. Soc. 1995, 117, 508

70. I. J. Dance. $\mathrm{Ti}_{8} \mathrm{C}_{12}$ : Barrierless Transformation of the $T_{h}$ Isomer to the $T_{d}$ Isomer. J. Am. Chem. Soc. 1996, 118, 6309

71. L. S. Wang, S. Li, and H. Wu. Photoelectron spectroscopy and electronic structure of met-car Ti8C12. J. Phys. Chem. 1966, 100, 19211

72. L. S. Wang and H. Cheng. Growth pathways of metallocarbohedrenes. Phys. Rev. Lett. 1997, 78,2983

73. J. S. Pilgrim and M. A. Duncan. Beyond metallo-carbohedrenes: Growth and decomposition of metal-carbon nanocrystals. J. Am. Chem. Soc. 1993, 115, 9724

74. J. S. Pilgrim and M. A. Duncan. Photodissociation of metallo-carbohedrene ("Met-Cars") cluster cations. J. Am. Chem. Soc. 1993, 115, 4395 
75. H. Sakurai and A. W. Castleman, Jr. Ionization potentials for the Titanium, Zirconium, and the mixed metal met-cars. J. Phys. Chem. A 1998, 102, 10486

76. Z. Y. Chen and A. W. Castleman, Jr. Growth of titanium nitride: From clusters to microcrystals. J. Chem. Phys. 1993, 98, 231.

77. K. Wade, J. Chem. Soc. D Chem. Commun. The structural significance of the number of skeletal bonding electron-pairs in carboranes, the higher boranes and borane anions, and various transition-metal carbonyl cluster compounds 1971, 792

78. D. M. P. Mingos, A General Theory for Cluster and Ring Compounds of the Main Group and Transition Elements. Nat. Phys. Sci. 1972, 236, 99;

79. D. M. P. Mingos, Polyhedral skeletal electron pair approach. Acc. Chem. Res. 1984, 17, 311.

80. X. Li,, A. Grubisic, S. T. Stokes, G. F. Gantefor, K. H. Bowen, K. Boggavarapu, M. Willis, P. Jena, R. Burgert, and H. Schnockel. Unexpected stability of $\mathrm{Al}_{4} \mathrm{H}_{6}$ : A borane analog? Science 2007, 315, 356.

81. A. Grubisic, X. Li, C. J. Stokes, G. F. Gantefor, K. Bowen, B. Kiran, P. Jena, R. Burgert, and H. Schnokel. Closo-alanes $\left(\mathrm{Al}_{4} \mathrm{H}_{4}, \mathrm{Al}_{\mathrm{n}} \mathrm{H}_{\mathrm{n}+2}(4 \leq \mathrm{n} \leq 8)\right)$ : A New Chapter in Aluminum Hydride Chemistry, J. Am. Chem. Soc. 2007, 129, 5969.

82. R. G. Wheeler, K. LaiHing, W. L. Wilson, and M. A. Duncan. Growth patterns in binary clusters of Group IV and V metals. J. Chem. Phys. 1988, 88, 2831.

83. R. W. Farley and A. W. Castleman, Jr. Observation of Gas-Phase Anionic Bismuth Zintl Ions. J. Am. Chem. Soc. 1989, 111, 2734.

84. A. E. Kuznetsov, A. I. Boldyrev, X. Li, and L. S.Wang, On the Aromaticity of Square Planar $\mathrm{Ga}_{4}{ }^{2-}$ and $\mathrm{In}_{4}{ }^{2-}$ in Gaseous $\mathrm{NaGa}_{4}{ }^{-}$and $\mathrm{NaIn}_{4}{ }^{-}$Clusters. J. Am. Chem. Soc.2001, 123, 8825.

85. L. F. Cui, X. Huang, L. M. Wang, D. Y. Zubarev, A. I. Boldyrev, J. Li, and L. S. Wang, $\mathrm{Sn}_{12}{ }^{2-}$ : Stannaspherene J. Am. Chem. Soc. 2006, 128, 8390.

86. L. F. Cui, X. Huang, L. M. Wang, J. Li, and L. S. Wang, $\mathrm{Pb}_{12}{ }^{2-}$ : Plumbaspherene. J. Phys. Chem. A2006, 110, 10169.

87. J.-P. Dognon, C. Clabaguera, and P. Pyykko, Towards a 32-Electron Principle: $\mathrm{Pu} @ \mathrm{~Pb}_{12}$ and Related Systems. Angew. Chem., Int. Ed. 2007, 46, 1427.

88. A. Grubisic, H. Wang, X. Li, Y.-J. Ko, F. S. Kocak, M. R. Pederson, K. H. Bowen, and B.W. Eichhorn. Photoelectron spectroscopic and computational studies of the Pt@Pb10 ${ }^{1-}$ and Pt@Pb12 ${ }^{1-2-}$ anions. Proc. Natl. Acad. Sci. U.S.A. 2011, 108, 14757. 
89. U. Rohrmann, S. Schaefer, and R. Schaefer, Size- and Temperature-Dependent Magnetic Response of Molecular Cage Clusters: Manganese-Doped Tin Clusters. J. Phys. Chem. A 2009, $113,12115$.

90. H. Wang, X. Zhang, Y. J. Ko, A. Grubisic, X. Li, G. Ganteför, H. Schnöckel,B. W. Eichhorn, M. S. Lee, P. Jena, A. K. Kandalam, B. Kiran, and K. H. Bowen. Aluminum Zintl anion moieties within sodium aluminum clusters. J. Chem. Phys. 2014, 140, 054301. 


\section{Graphical Abstract}
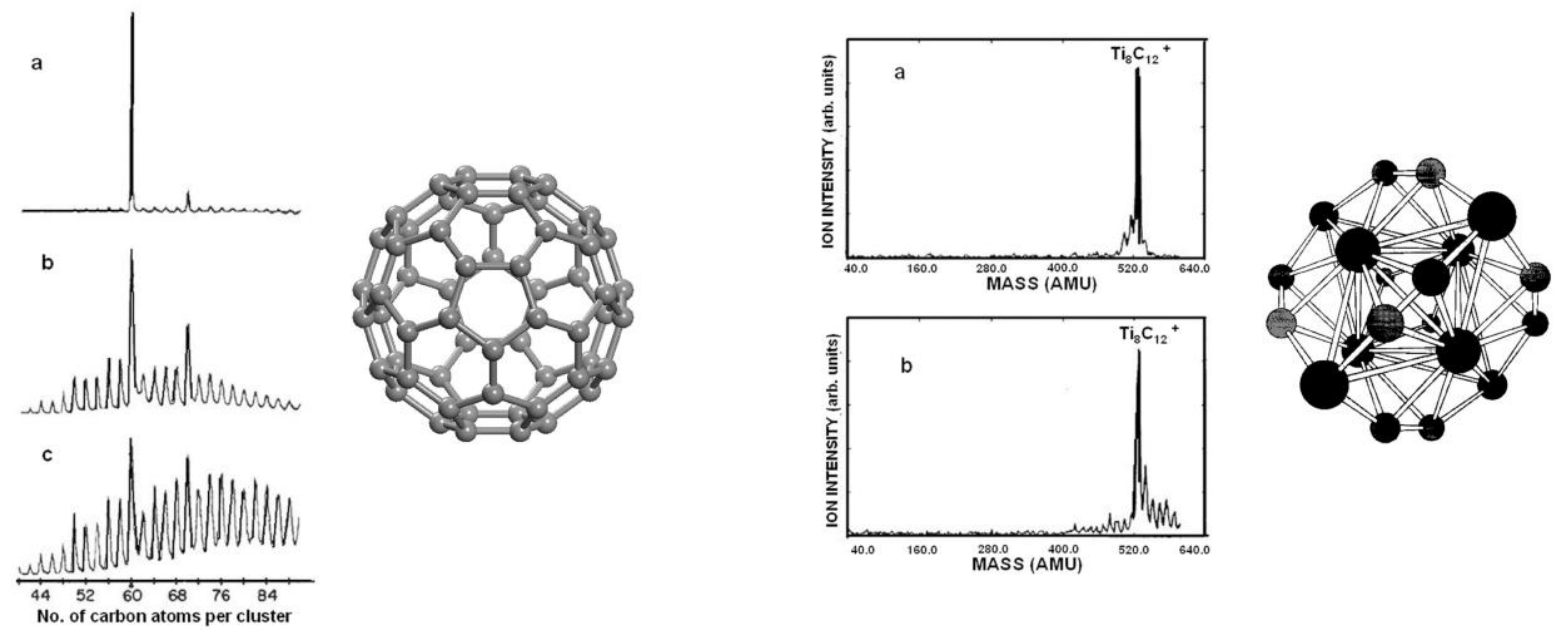

Mass spectra of $\mathrm{C}_{60}$ and $\mathrm{Ti}_{8} \mathrm{C}_{12}$ and corresponding structures. 